This item was submitted to Loughborough's Research Repository by the author.

Items in Figshare are protected by copyright, with all rights reserved, unless otherwise indicated.

\title{
An estimation of distribution algorithm with filtering and learning
}

PLEASE CITE THE PUBLISHED VERSION

https://doi.org/10.1109/tase.2020.3019694

\section{PUBLISHER}

Institute of Electrical and Electronics Engineers (IEEE)

\section{VERSION}

AM (Accepted Manuscript)

\section{PUBLISHER STATEMENT}

(c) 2020 IEEE. Personal use of this material is permitted. Permission from IEEE must be obtained for all other uses, in any current or future media, including reprinting/republishing this material for advertising or promotional purposes, creating new collective works, for resale or redistribution to servers or lists, or reuse of any copyrighted component of this work in other works.

\section{LICENCE}

All Rights Reserved

\section{REPOSITORY RECORD}

Tang, Lixin, Xiangman Song, Jiyin Liu, and Chang Liu. 2020. "An Estimation of Distribution Algorithm with Filtering and Learning”. Loughborough University. https://hdl.handle.net/2134/14245547.v1. 


\title{
An Estimation of Distribution Algorithm with Filtering and Learning
}

\author{
Lixin Tang, Senior Member, IEEE, Xiangman Song, Jiyin Liu, Chang Liu
}

\begin{abstract}
Estimation of distribution algorithm (EDA) is an efficient population-based stochastic search technique. Since it was proposed, many attempts have been made to improve its performance in the context of non-linear continuous optimization. However, the success of EDA depends on the accuracy of modeling, the effectiveness of sampling and the ability of exploration. An effective EDA often needs to take some measures to adjust the model and to guide sampling. In this paper, we propose a novel estimation of distribution algorithm which applies the idea of Kalman filtering to revise the modeling data and a learning strategy to improve sampling. The filtering scheme modifies the modeling data set using an estimation error matrix based on historic solution data. During the sampling process, the learning strategy determines the region to sample next based on the sampling outcomes so far, instead of completely random sampling. The proposed EDA also employs a multivariate probabilistic model based on copula function and can quickly reach the promising area in which the optimal solution is likely to be located. A collection of general benchmark functions are used to test the performance of the proposed algorithm. Computational experiments show that the EDA is effective.
\end{abstract}

Note to Practitioners - In many process industries, there exist black-box operation optimization problems and large scale nonlinear optimization problems with variable coupling. For these problems, it is difficult to establish mechanism models between input and output. However, real-time data can be measured from the system through sensors. We can utilize this process information to optimize the system so as to attain the desired objective. In this paper, we propose a novel estimation of distribution algorithm which applies a filtering scheme to revise the modeling data and a learning strategy to improve sampling, which can solve the problems with the characteristics of nonlinearity, variable coupling and large scale. Computational experiments show that the EDA is effective. In the future, the proposed algorithm can be applied to some practical optimization problems such as operation optimization in blast furnace, which is considered as a continuous

This work was supported by the National Key Research and Development Program of China (2016YFB0901900), the Major International Joint Research Project of the National Natural Science Foundation of China (71520107004), the Major Program of National Natural Science Foundation of China (71790614), the Fund for Innovative Research Groups of the National Natural Science Foundation of China (71621061), and the 111 Project (B16009).

Lixin Tang is with Key Laboratory of Data Analytics and Optimization for Smart Industry (Northeastern University), Ministry of Education, 110819 China (e-mail: lixintang@mail.neu.edu.cn).

Xiangman Song is with Liaoning Engineering Laboratory of operation Analytics and Optimization for Smart Industry, State Key Laboratory of Synthetical Automation for Process Industries, Northeastern University, Shenyang 110819, China (e-mail: sxm123121@163.com).

Jiyin Liu is with School of Business and Economics, Loughborough University, Leicestershire LE11 3TU, UK (e-mail: J.Y.Liu@lboro.ac.uk).

Chang Liu is with Liaoning Key Laboratory of Manufacturing System and Logistics, Northeastern University, Shenyang 110819, China (e-mail: 1c1987328@126.com). production process with variable coupling. The algorithm has the potential to help optimizing the process control parameters.

Index Terms - estimation of distribution algorithm, filtering, learning sampling, multivariate probabilistic model.

\section{INTRODUCTION}

JNELLIGENT algorithms have become very popular for $\mathbf{I}_{\text {solving optimization problems and are studied widely in the }}$ practical problems [1-3]. Estimation of distribution algorithms (EDAs) are evolutionary algorithms based on estimation and sampling from probabilistic models. The first EDA was developed by Mühlenbein and Paaß [4], and several improved EDAs have been proposed in recent years. Gaussian distribution is commonly used for modeling in continuous EDAs. $\mathrm{UMDA}_{c}{ }^{\mathrm{G}}$ [5], the earliest proposed univariate Gaussian-based $\mathrm{EDA}$, is easy to implement due to its ignoring the dependency of all variables. But it is difficult to solve problems in which the variables have strong dependency with each other. EMNAglobal [5] was proposed to overcome this. It used a conventional maximum likelihood estimated multivariate Gaussian distribution. Later, EEDA [6] was proposed to improve the poor explorative capability. Besides, EDAs based on complex Gaussian distribution are proposed to solve multimodal and intractable problems. Some are with Gaussian mixture distribution [7-9]. Considering premature convergence of the traditional Gaussian EDAs, EDA ${ }^{2}$ was proposed to deal with it [10]. EDAs have also been used in hybrid algorithms. An algorithm combining DE and EDA was proposed for the global continuous optimization problem [11], and it used an offspring generation scheme, which was similar to the DE crossover. For large-scale optimization problems, EDA-MCC [12] performed well using a specially designed multivariate model. Gaussian models can furnish useful information to search the optimal solution, but they cannot always provide an accurate distribution of promising solutions.

Increasingly, EDAs based on different models have been studied. With the capacity of describing arbitrary multimodality, the Histogram models in EDAs [13-17] are more flexible than Gaussian models. However, the complexity can be increased with problem size on account of multiple variable dependencies [18]. Some EDAs can learn the structure and parameters of the model in each generation. The factorized distribution algorithm (FDA) [19] can extract a factorization structure from a priori knowledge of the problem. EDAs based on Bayesian networks [20] using directed acyclic graphical models can update local parameters according to the condi- 
tional probability distributions. Based on Markov random fields, DEUM [21] presented a fitness modeling approach to estimating the parameters of the model. Clustering techniques [22] and other statistical methods, such as some variants of the expectation-maximization (EM) algorithm [23], have been used to learn mixtures of distributions in EDAs. These EDAs have given greater emphasis to the modeling structure. But the frameworks of these EDAs are all similar and the historic individual information has not been utilized fully.

In recent years, EDAs have been proposed to deal with many practical problems. A Pareto-Based EDA was developed to solve a multi-objective flow-shop scheduling problem [24]. Using a mixed probability distribution model and an adaptive scheme, a new EDA was proposed to solve a multipolicy insurance investment problem [25].

In this paper, a novel Estimation of Distribution Algorithm with Filtering and Learning (EDA-FL) is proposed to solve continuous optimization problems more effectively. The contributions of our proposed algorithm are as follows:

1) A new EDA framework incorporating filtering is proposed. The idea of Kalman filtering is adopted to modify some individuals, which in turn affect the model, using an estimation error matrix from historic information.

2) A sampling strategy with learning is proposed. Using this strategy, the decision on where to sample is made according to the statistical information about the objective values of sampled individuals to avoid completely random sampling.

To illustrate the effectiveness, the proposed algorithm is tested through computational experiments on benchmark functions.

The rest of this paper is organized as follows. Section II presents the background knowledge, including EDA, copulas and Kalman filtering. Section III describes the fundamental ideas of the EDA-FL algorithm. Experimental studies are shown in Section IV. Finally, conclusions are given in section V.

\section{BACKGROUND}

\section{A. Estimation of Distribution Algorithm}

Estimation of Distribution Algorithm (EDA) is an evolutionary algorithm that uses a probability model based on the distribution of good individuals in the current population to generate individuals of the next generation. The probability model also changes as the population evolves in the search process. Unlike in other evolutionary algorithms, in EDA, each individual in the new generation is not generated by mutation and crossover operations on the individuals of the old generation. Instead, the evolution is at the "macro" level. So, EDA emphasizes on the global information in the search process and has a strong exploration capability.

EDA works iteratively, as shown in Fig. 1, where Pop represents population or a set of individuals. In this paper, we use bold capital letters for matrices or populations, e.g., $\mathbf{A}$ and Pop $^{\text {mod }}$. Vectors and individuals are represented by lowercase bold characters, e.g., $\mathbf{u}$ and $\mathbf{x}_{\text {best. }}$ The main steps of EDA are described as follows.

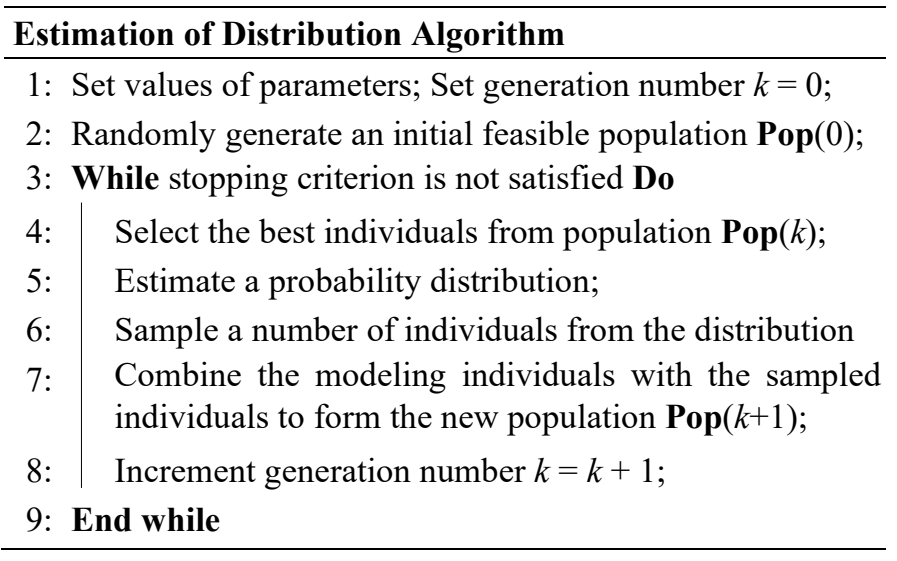

Fig.1. Pseudo-code of Estimation of Distribution Algorithm

1) Selection: Selection aims to obtain a set of good individuals from the current population. It is expected that the selected individuals can describe the solution space accurately and comprehensively. The most widely used selection method is the truncation selection.

2) Modeling: Using a statistical method, the probabilistic model is constructed based on the relevant statistical information extracted from the selected individuals.

3) Sampling: In the sampling step, a set of individuals called sampled individuals is generated from the constructed probabilistic model. The sampling method is dependent on the modeling method used.

4) Combination: In every generation, the offspring population is often obtained by combining the modeling individuals and sampled individuals. In addition, individuals generated randomly according to the uniform distribution in the search space can also be added into the offspring population. The commonly used stopping criterion is the number of Fitness Evaluations ( $n-F E s)$ reaching a predefined maximum value.

\section{B. Copulas}

A concise definition of copulas is given in [26]: A copula is a multivariate probabilistic distribution function $C(\bullet)$ of $D$ dimensional random vector $\mathbf{u}=\left(u_{1}, \ldots, u_{D}\right)$ on the unit hypercube $[0,1]^{D}$ with some properties. As can easily be seen, the most well-known copula is the independence distribution function:

$$
C\left(u_{1}, \ldots, u_{n}\right)=\prod_{i=1}^{n} u_{i}
$$

There are two important copulas, which are interesting in practical applications, namely, Gaussian-copula and empirical-copula. Their marginal distributions are Gaussian and empirical distributions respectively. Other copulas include $t$-copula, Clayton-copula, Gumbel-copula and Frank-copula, etc. [26].

The key theorem was introduced by Sklar [27] to separate the effect of dependence from the effect of marginal distributions in a copula.

For a $D$-dimensional random vector $\mathbf{x}=\left(x_{1}, x_{2}, \ldots, x_{D}\right)$, its joint distribution function being $F(\mathbf{x})$ with the marginal distri- 
butions $u_{d}=F_{d}\left(x_{d}\right), d=1, \ldots, D$, there exists a copula $C(\mathbf{u})$, which is a multivariate distribution function of vector $\mathbf{u}=\left(u_{1}\right.$, $\left.u_{2}, \ldots, u_{D}\right)$ in $[0,1]^{D}$. The following equality was set up and the proof was given in [27].

$$
\begin{aligned}
C\left(u_{1}, \ldots, u_{d}\right) & =F\left(x_{1}, \ldots, x_{d}\right) \\
& =F\left(F_{1}^{-1}\left(u_{1}\right), \ldots, F_{d}^{-1}\left(u_{d}\right)\right)
\end{aligned}
$$

Where $F_{d}^{-1}\left(u_{d}\right)=\sup \left\{x_{d} \mid F_{d}\left(x_{d}\right) \leq u_{d}\right\}$

With the analysis above, each $\mathbf{x}=\left(x_{1}, x_{2}, \ldots, x_{D}\right)$ can be generated in two steps. The first step is to generate a random vector $\mathbf{u}=\left(u_{1}, u_{2}, \ldots, u_{D}\right)$ with the copula function. In the second step, vector $\mathbf{x}=\left(x_{1}, x_{2}, \ldots, x_{D}\right)$ is generated using the common inverse function of the marginal distribution of each variable, i.e., $x_{d}=F_{d}^{-1}\left(u_{d}\right), d=1, \ldots, D$.

In recent years, many EDAs using copulas as probabilistic models (copula-EDAs) have been proposed. Based on the copula theory, a framework of EDA was proposed in [28] to solve two-dimensional optimization problems. Archimedean-copula, Gumbel-copula and Gaussian-copula have all been used in multivariate EDAs [29-33], which were tested on problems of low dimensions in small scale experiments. The Maximum Likelihood Estimation was used in a copula-EDA to estimate the parameters of copula [34]. An EDA based on Gaussian copulas was proposed in [35] to solve multi-objective problems and applied to RFID network planning. An EDA using multivariate extension of the Archimedean copula was presented in [36]. A hybrid algorithm was proposed in [37] combining Archimedean copula based EDA and artificial bee colony algorithm to achieve faster convergence. A new EDA based on parallel copula was also proposed to improve the efficiency [38]. These algorithms were tested on a small number of benchmark functions. To deal with the major drawbacks, including too much emphasis on parameters and premature convergence, a new EDA based on Multivariate Elliptical Copulas was presented, where the parameters were estimated and a population diversity technique was used [39]. EDAs have also been applied to solve problems in practical settings. For example, an EDA was proposed in [40] to solve problems involving various types of constraints, while EDAs were applied in $[41,42]$ to solve flow-shop scheduling problems.

\section{Kalman Filtering Process}

Kalman filtering [43] is a recursive estimator that uses a series of predictions and observations to make estimation of unknown variables.

For a discrete-time linear system, Kalman filtering model is used to predict the true state $\mathbf{x} \in \square^{n}$ at time $k$ from the state at $(k-1)$ according to a linear stochastic difference equation,

$$
\mathbf{x}(k)=\mathbf{A x}(k-1)+\mathbf{B} \boldsymbol{\theta}(k)+\mathbf{w}(k)
$$

At time $k$ an observation $\mathbf{y} \in \square^{m}$ of the true state is given by

$$
\mathbf{y}(k)=\mathbf{H x}(k)+\mathbf{v}(k)
$$

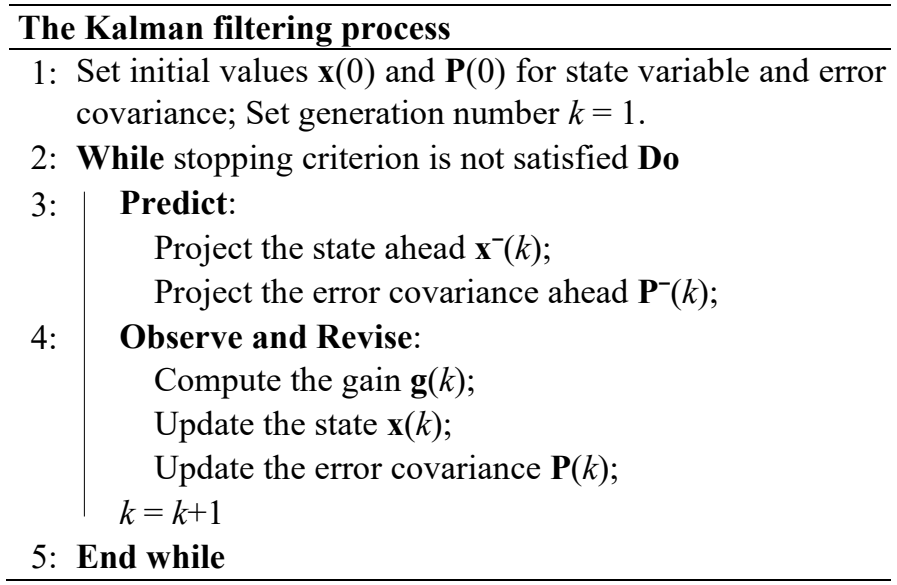

Fig.2. The Kalman filtering process

Where $\mathbf{A}$ is the state-transition matrix which is applied to the previous state, $\mathbf{B}$ is the co-efficient matrix for the control input $\boldsymbol{\theta} \in \square^{l}, \mathbf{H}$ is known as the observation matrix, $\mathbf{w}(k)$ is the process noise, and $\mathbf{v}(k)$ is observation noise.

The Kalman filtering process can be described in Fig. 2, where $\mathbf{x}^{-}(k)$ is the predicted state, $\mathbf{P}^{-}(k)$ is the predicted error covariance, $\mathbf{g}(k)$ is the Kalman gain, $\mathbf{x}(k)$ is the revised state, $\mathbf{P}(k)$ is the posteriori error covariance. $\mathbf{x}(0)$ and $\mathbf{P}(0)$ are the initial values of the state variable and the error covariance, respectively.

In this paper, we construct the new EDA framework through prediction, sampling and revision operations using the idea of Kalman filtering.

\section{The Proposed ALgORITHM}

\section{A. A New EDA Framework Incorporating Filtering}

In essence, an optimization problem can be viewed as a system. The solution process of the optimization problem can then be considered as the process of searching for the ideal state of the system guided by observation, for which Kalman filtering is an effective technique.Tan et al. [44] have proposed a new dynamic multi-objective optimization evolutionary algorithms using Kalman filtering, in which a prediction model is designed to learn patterns from past data. In our new EDA, we transform the best individuals in the last population to make predictions of the optimal solution. A set of the best individuals among those in the last population as well as the predicted individuals are then used for modeling. The samples generated can be considered as observed solutions. To improve the accuracy of the solution, having been inspired by Kalman filtering process, we revise some individuals based on the difference between the predicted and observed data. The revision in turn affects the set of individuals for modeling in the next generation (iteration). Hopefully this can improve the effectiveness of the model and thus the EDA process. Our new EDA framework with filtering can be outlined as Fig. 3. In the framework, $\operatorname{Pop}(k)$ is the population of iteration $k$; $\mathbf{P o p}^{\text {mod }}(k), \mathbf{P o p}^{\text {sam }}(k), \mathbf{P o p}^{\text {pre }}(k)$ and $\mathbf{P o p}^{r e v}(k)$ are the sets of modeling, sampled, predicted and revised individuals, respectively. The names of functions are in capitals and their meanings are obvious. 


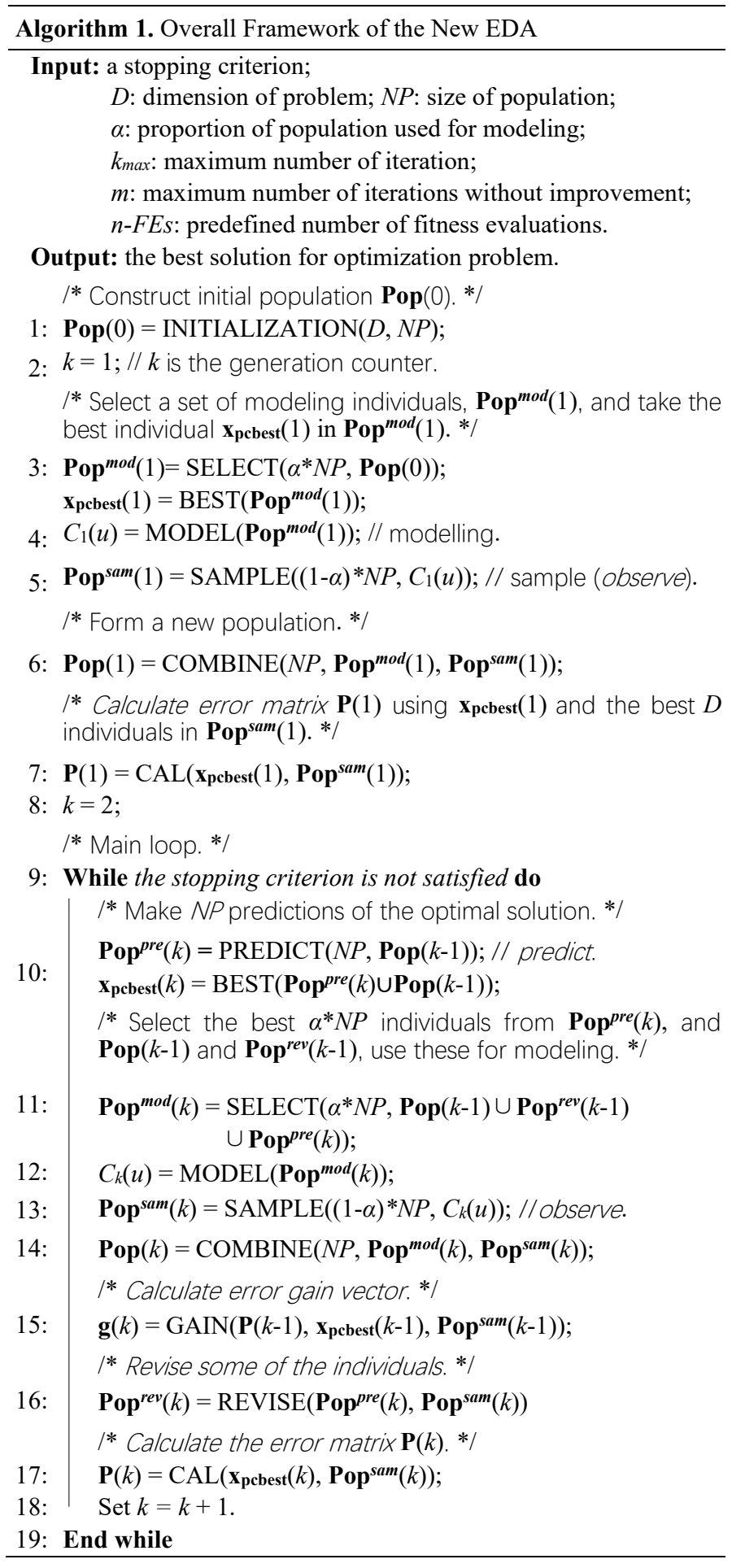

Fig.3. The new EDA framework with filtering

In each iteration of this framework, the basic EDA steps are listed in normal font. In this paper we introduce a learning strategy to improve sampling step. The details of the strategy will be presented in the next subsection. We incorporate the idea of Kalman filtering in the framework and the steps related to filtering are indicated in italic. The EDA search process is clearly not a linear system, thus the same linear transformations used in Kalman filtering for linear systems cannot be directly applied. By defining new error matrix and gain vector based on individuals and their objective values in the EDA process, we propose to use different form of transformation (still keeping linear) here to achieve similar purpose to those in linear systems. The filtering steps embedded in the EDA framework include prediction, observation and updating of the gain vector and error matrix. Details are given below in the description of the relevant steps.

1) Initialization and the first iteration: The function $\operatorname{Pop}(0)$ $=$ INITIALIZATION $(D, N P)$ in Step 1 of the framework initializes the algorithm, which is generating an initial population of $N P$ individuals by drawing the $D$-dimension variable values randomly from uniform distributions over their search ranges. The algorithm then starts iterations.

The first iteration $(k=1)$ is presented separately in the framework (steps 2 -7) because some filtering operations need information from previous iteration and so need to be done differently or cannot be done in the first iteration. The main operations in later iterations $(k>1)$ are presented in steps 9 - 19 of the framework. The first iteration of the new EDA framework includes two filtering operations, observation (in step 5) and error matrix $\mathbf{P}(k)$ calculation (step 7), which enable the other filtering operations in the next iterations.

To calculate $\mathbf{P}(k)$, we first find the best individual $\mathbf{x}_{\text {pcbest }}(k)$ in the population using function $\operatorname{BEST}(\bullet)$ (steps 3 and 10), and select the best $D$ individuals among the sampled ones. $\mathbf{P}(k)$ is then calculated as a $D \times D$ matrix with each row being the difference between one of these $D$ individuals and the best individual $\mathbf{x}_{\text {pcbest }}(k)$.

$$
\begin{aligned}
\mathbf{P}(k) & =\left[\begin{array}{ccc}
p_{11}(k) & \ldots & p_{1 D}(k) \\
\ldots & \ldots & \ldots \\
p_{D 1}(k) & \ldots & p_{D D}(k)
\end{array}\right] \\
& =\left[\begin{array}{ccc}
x_{p c b e s t, 1}(k)-z_{1,1}(k) & \ldots & x_{p c b e s t, D}(k)-z_{1, D}(k) \\
\ldots & \ldots & \ldots \\
x_{p c b e s t, 1}(k)-z_{D, 1}(k) & \ldots & x_{p c b e s t, D}(k)-z_{D, D}(k)
\end{array}\right]
\end{aligned}
$$

where $\mathbf{z}_{\mathbf{i}}(k)=\left[z_{i, 1}(k), z_{i, 2}(k), \ldots, z_{i, D}(k)\right], i=1, \ldots, D$, are the best $D$ sampled individuals in $\operatorname{Pop}^{\text {sam }}(k)$. In this step, the error matrix $\mathbf{P}$ is calculated in $O\left(D^{2}\right)$ time.

2) Prediction (step 10): The optimal solution is predicted by transforming the best individual in the population $\operatorname{Pop}(k-1)$ plus some noise represented by the difference of two randomly selected individuals in the population. This is expressed in formula (6). Function PREDICT(NP, Pop $(k-1))$ in Fig. 3 generates NP predicted solutions in this way and stores them in $\operatorname{Pop}^{\text {pre }}(k)$.

$$
\mathbf{x}_{\mathbf{i}}^{-}(k)=\mathbf{A} \mathbf{x}_{\text {best }}(k-1)+\boldsymbol{\Lambda}\left[\mathbf{x}_{\mathbf{a}}(k-1)-\mathbf{x}_{\mathbf{b}}(k-1)\right]
$$

where $\mathbf{x}_{\mathbf{i}}^{-}(k), i=1, \ldots, N P$, is a predicted solution. $\mathbf{A}$ is identity matrix, $\mathbf{x}_{\text {best }}(k-1)$ is the best individual, $\mathbf{x}_{\mathbf{a}}(k-1)$ and $\mathbf{x}_{\mathbf{b}}(k-1), a \neq$ $b$, are two random individuals in the last population $\operatorname{Pop}(k-1)$. To guarantee diversity of predictions, $\boldsymbol{\Lambda}$ is set to be a diagonal 


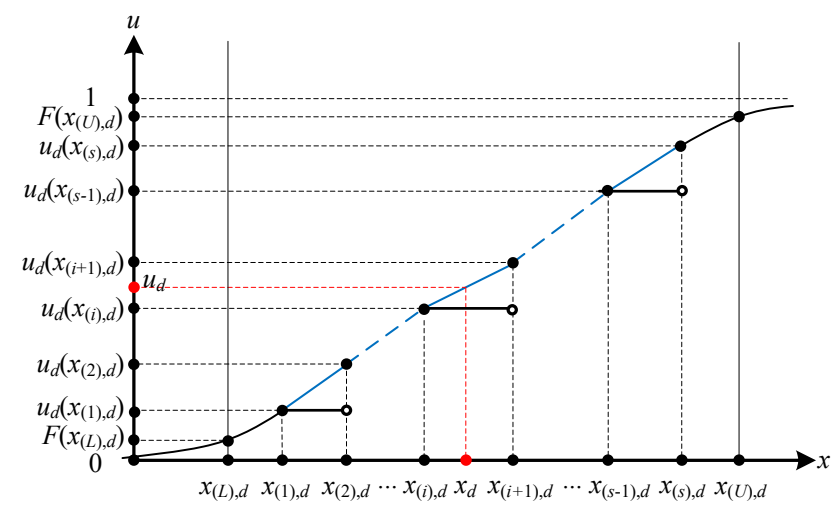

Fig.4. Obtaining $x_{d}$ from $u_{d}$ using inverses of the distribution

matrix. $p_{0}$ proportion of the diagonal elements of $\boldsymbol{\Lambda}$ are randomly generated from the uniform distribution $U[0,1]$, while all the other diagonal elements are the same with a value between 0 and 1. Each of $N P$ predicted solutions needs $O(D)$ steps for generating $D$ elements, so this is done in $O\left(D^{*} N P\right)$ time.

3) Selection: In steps 3 and 11 , the $\alpha$ proportion $(\alpha<1)$ of the best individuals is selected according to their objective function values. Hence, $s=\alpha^{*} N P$ denotes the number of the selected individuals for modeling. In step 11 (iteration 2 onwards), the best $\alpha^{*} N P$ are selected among individuals in the population as well as the revised and predicted individuals.

4) Modeling (steps 4 and 12): Modeling is designed to establish the probability distribution of optimal solution using the selected set of solutions. For all individuals $\mathbf{x}_{\mathbf{i}}=\left(x_{i, 1}, x_{i, 2}, \ldots\right.$, $\left.x_{i, D}\right), i=1, \ldots, s$, in $\mathbf{P o p}^{\text {mod }}$, we first sort their elements in each dimension $d$ and denote the $i^{\text {th }}$ smallest element in dimension $d$ as $x_{(i), d}, i=1, \ldots, s ; d=1, \ldots, D$. With this information we then calculate $\mathbf{u}=\left(u_{1}, \ldots, u_{d}, \ldots, u_{D}\right)$ as follows. Fig. 4 shows the marginal distribution $F_{d}\left(x_{d}\right)$ being a mixture of empirical distribution and Gaussian distribution, which is a piece-wise function. This model allows sample individuals to be outside the area of modeling individuals, which enhances the ability of exploration.

$$
\begin{aligned}
& u_{d}\left(x_{(i), d}\right)=F\left(x_{(1), d}\right)+(i-1) *\left[F\left(x_{(s), d}\right)-F\left(x_{(1), d}\right)\right] /(s-1), \\
& i=1, \ldots, s \\
& F\left(x_{(\triangleright, d}\right)=P\left(x \leq x_{(\triangleright), d}, x \sim N\left(\bar{x}, \sigma^{2}\right)\right), \\
& x_{(Ð), d} \leq x_{(1), d} \text { or } x_{(\triangleright, d} \geq x_{(s), d}
\end{aligned}
$$

Each selected individual corresponds to a point in $u$ space. Meanwhile, we divide interval $[0,1]$ into $K$ partitions $S_{1}, \ldots, S_{K}$, and the length of each partition $\delta=1 / K$. So there are $K^{D}$ subcubes. Then the copula density function is defined as

$$
c\left(u_{1}, \ldots, u_{D}\right)=N_{j} / s / \delta^{D}, j \in\left\{S_{1}, \ldots, S_{K}\right\}^{D}
$$

where $N_{j}$ denotes the number of points in certain subcube $j$. As remarked in [45], this step has a time complexity of $\mathrm{O}\left(D^{*} s^{*} \log s\right)$.

5) Sampling (steps 5 and 13): The distribution model in the last step is used to generate $(1-\alpha) * N P$ individuals. This sam- pling operation of EDA here also serves as observation operation for filtering. The objective function value of a sampled individual, $f(\mathbf{x})$, can be viewed as a value of the observation variable $\mathbf{y}$ in Kalman filtering. In this paper, the method of an interpolation is used as the inverses of the distribution to generate the individual $\mathbf{x}=\left(x_{1}, x_{2}, \ldots, x_{D}\right)$. We first generate a vector $\mathbf{u}=\left(u_{1}, u_{2}, \ldots, u_{D}\right)$. Here $u_{1}$ is a random variate from the uniform distribution on interval $[0,1]$, while $u_{d}, d=2, \ldots, D$, is generated with the conditional distribution function $C_{d}\left(u_{d} \mid u_{1}, \ldots\right.$, $\left.u_{d-1}\right)$ considering the relation $C_{d}\left(u_{d} \mid u_{1}, \ldots, u_{d-1}\right)=u_{d}{ }^{*}$, where $u_{d}{ }^{*}$, $d=2, \ldots, D$, is a random number generated from the uniform distribution on $(0,1]$. For a generated $u_{d}$ that falls in the interval between $u_{d}\left(x_{(i), d}\right)$ and $u_{d}\left(x_{(i+1), d}\right)$, the corresponding $x_{d}$ is in the interval between $x_{(i), d}$ and $x_{(i+1), d}, i=1, \ldots, s-1$, and can be calculated using interpolation as follows.

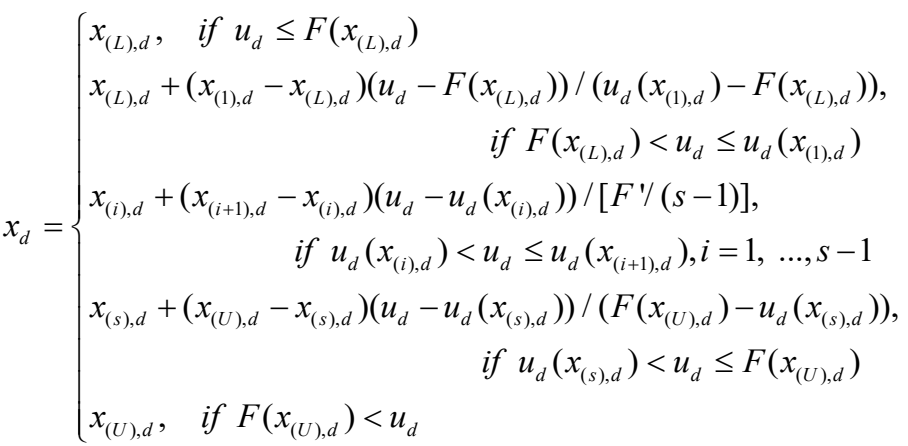

where $F^{\prime}=u_{d}\left(x_{(s), d}\right)-u_{d}\left(x_{(1), d}\right)$

In this step, a random vector $x$ is generated in $O\left(D^{*} \log K\right)$ time. The sampled individuals are then combined with the modeling individuals to form a new population (steps 6 and 14).

6) Calculating the gain vector (function $\operatorname{GAIN}(\cdot)$ in step $15)$ : The gain vector $\mathbf{g}(k)$ is a $D$-dimensional vector with each element being the average error weight of an individual in a specific dimension relative to the error of the objective value. Using $\mathbf{P}(k-1), g_{d}(k), d=1, \ldots, D$, is calculated as follows.

$g_{d}(k)=\frac{\sum_{i=1}^{D}\left(P_{i, d}(k-1)\right)}{\sum_{d^{\prime}=1}^{D} \sum_{i=1}^{D}\left|P_{i, d^{\prime}}(k-1)\right| \sum_{i=1}^{D}\left(f\left(\mathbf{z}_{\mathbf{i}}(k-1)\right)-f\left(\mathbf{x}_{\text {pcbest }}(k-1)\right)\right) / D}$

where $\mathbf{P}(k-1)$ is the error matrix, $f\left(\mathbf{z}_{\mathbf{i}}(k-1)\right)$ and $f\left(\mathbf{x}_{\text {pcbest }}(k-1)\right)$ are the objective values of $\mathbf{z i}(k-1)$ and $\mathbf{x}_{\text {pcbest }}(k-1)$ respectively. This step has a time complexity of $O(D)$.

7) Revision: Employing the error gain vector, the revised individuals can be obtained in step 16 (function $\operatorname{REVISE}(\bullet)$ ) using formulae (11), (12) and (13). The meaning of the above defined gain vector makes the revision operation linear.

$$
\begin{gathered}
\widehat{\mathbf{x}}_{\mathbf{i}}(k)=\mathbf{x}_{\mathbf{i}}(k)+\mathbf{g}(k)\left[f\left(\mathbf{z}_{\text {best }}(k)\right)-f\left(\mathbf{x}_{\mathbf{i}}(k)\right)\right] \\
\widehat{\mathbf{x}}_{\text {pcbest }}(k)=\mathbf{x}_{\text {pcbest }}(k)+\mathbf{g}(k)\left[f\left(\mathbf{z}_{\text {best }}(k)\right)-f\left(\mathbf{x}_{\text {pcbest }}(k)\right)\right]
\end{gathered}
$$




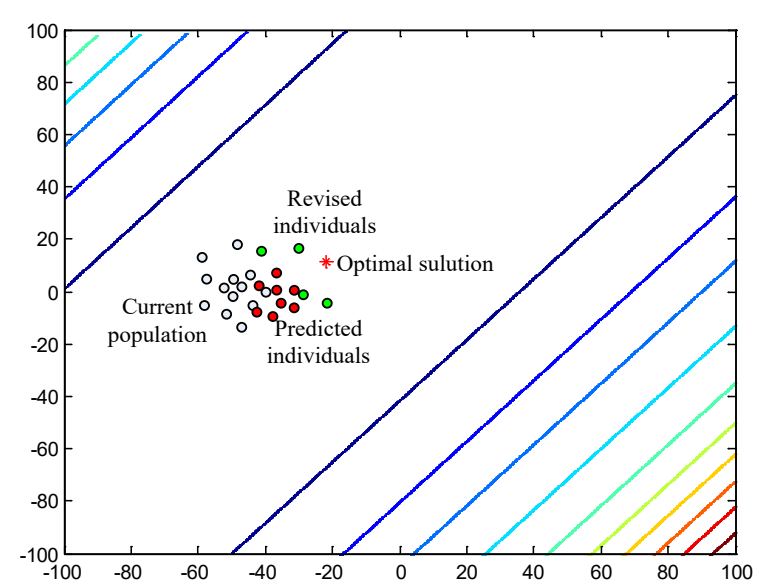

Fig.5. The influence on modeling individuals by predicting and revising

$$
\widehat{\mathbf{z}}_{\mathbf{i}}(k)=\mathbf{z}_{\mathbf{i}}(k)+\mathbf{g}(k)\left[f\left(\mathbf{x}_{\text {pcbest }}(k)\right)-f\left(\mathbf{z}_{\mathbf{i}}(k)\right)\right]
$$

where $\mathbf{x}_{\mathbf{i}}(k), i=1, \ldots, N P_{1}$, is the best $N P_{1}$ individuals among the predicted individuals and those in the population $\operatorname{Pop}(k-1) . \mathbf{Z} \mathbf{i}(k)$, $i=1, \ldots, N P_{2}$, is the best $N P_{2}$ individuals of the sampled individuals, Zzest $(k)$ is the best sampled individual. $N P_{1}=p_{1} * \alpha * N P$ and $N P_{2}=p_{2} * \alpha^{*} N P$ are determined by selecting the values of parameters $p_{1}(<1)$ and $p_{2}(<1) . \widehat{\mathbf{x}}_{\mathbf{i}}(k), \widehat{\mathbf{x}}_{\text {pcbest }}(k)$ and $\widehat{\mathbf{z}}_{\mathbf{i}}(k)$ are the revised individuals of $\mathbf{x}_{\mathbf{i}}(k), \mathbf{x}_{\text {pcbest }}(k)$ and $\mathbf{z}_{\mathbf{i}}(k)$, respectively. $\mathbf{x}_{\text {pebest }}(k)$ is obtained in steps 3 and 10 of Algorithm 1. Formula (12) is a special case of (11). The calculations in (11) and (13) generate the revised individuals in $O\left(N P_{1} * D+N P_{2} * D\right)$ time.

8) Calculating error matrix: The error matrix is calculated in step 17 (function CAL(•)), using formula (5).

Overall, in the new EDA framework, we introduced two distinct operations "Predict" and "Revise" which do not exist in the traditional EDA. The potential advantage of this is illustrated in Fig. 5, where the red asterisk represents the optimal solution. As iteration goes on, the individuals in the population may tend to concentrate in a small area. In this case it is difficult for the traditional EDA to evolve further even though the optimal solution is still far away from this area. The prediction and revision operations in the new EDA generate new solutions. The predicted and revised individuals as illustrated in Fig.5, not only tend to be closer to the optimal solution but also increase the diversity of the population and so makes it evolve further.

\section{B. Learning Sampling Strategy}

The process of EDA takes a long time to reach a good final solution if the sampling is completely random. To search the solution space more efficiently, we use the idea of optimal learning, see [46], in the sampling process of each generation, i.e., after an initial set of individuals is sampled randomly, each new individual is sampled in a promising region which is determined using the existing individuals. Using learning techniques to improve optimization process becomes popular in recent years. These techniques often consider the special features of the optimization problem being solved. For example, in the process of solving a pre-marshaling problem in [47], a learning model is used to choose the next move. We use opti-

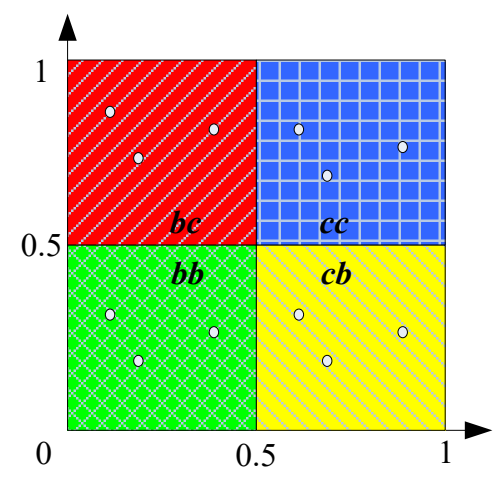

Fig.6. The four selections of $\left(u_{1}^{*}, u_{2}^{*}\right)$

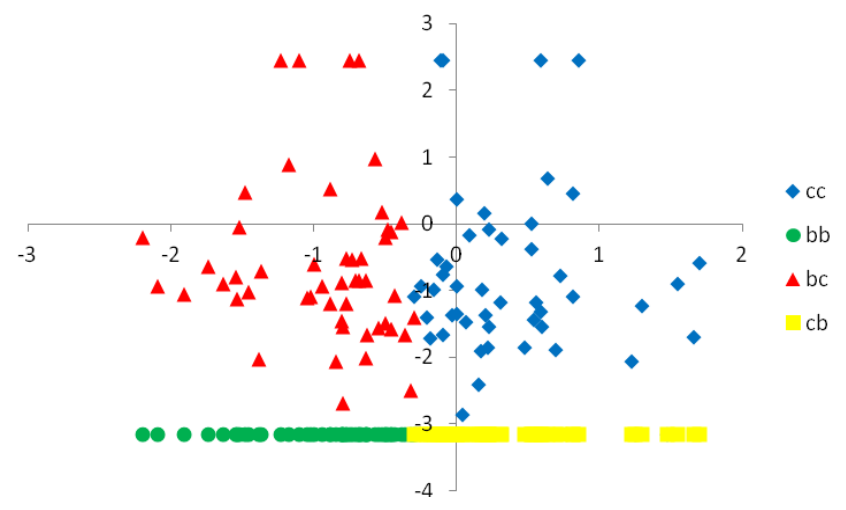

Fig.7. The sampling individuals $\left(x_{1}, x_{2}\right)$ in the different regions

mal learning to improve the sampling process of EDA as a general optimization method.

In the EDA based on copula function, because of the randomization of $u_{1}$ and $u_{d}{ }^{*}, d=2, \ldots, D$, we need sample large number of individuals to get a sufficiently good solution. Trying to improve this, we divide the whole sampling range of each dimension into multiple intervals, from which the vector $\mathbf{u}^{*}=$ $\left(u_{1}{ }^{*}, u_{2}{ }^{*}, \ldots, u_{D}{ }^{*}\right)$ can be generated, where $u_{1}{ }^{*}$ is defined as $u_{1}$ for convenience. Now we divide the range $[0,1]$ into $M$ equal intervals. So the length $L$ of each interval equals $1 / M$. Then $u_{d}{ }^{*}$ can be generated from $M$ alternative intervals $((j-1) \times L, j \times L], j=$ $1, \ldots, M$. Note that the first interval for $u_{1}$ also includes 0 . Consider a simple example with $D=2, M=2$, denote $b:=(0$, $0.5]$ and $c:=(0.5,1]$. So $\left(u_{1}{ }^{*}, u_{2}{ }^{*}\right)$ can be obtained from four alternative regions: $b b, b c, c b$ and $c c$, as shown in Fig. 6. We can select each region for $\left(u_{1}{ }^{*}, u_{2}{ }^{*}\right)$ and then sample individuals accordingly. Fig. 7 shows the individuals generated when $\left(u_{1}{ }^{*}\right.$, $\left.u_{2}{ }^{*}\right)$ are sampled from different regions. The axes in Fig.7 represent the decision variables for this example, $x_{1}$ and $x_{2}$. Suppose the objective function is $x_{1}^{2}+x_{2}^{2}$, Fig.7 shows that the individuals generated with $\left(u_{1}{ }^{*}, u_{2}{ }^{*}\right)$ sampled from $b c$ and $c c$ are better than those with $\left(u_{1}{ }^{*}, u_{2}{ }^{*}\right)$ from $b b$ and $c b$. So it will be beneficial to find a learning strategy such that promising regions of $u^{*}$ can be identified and individuals are generated accordingly. 


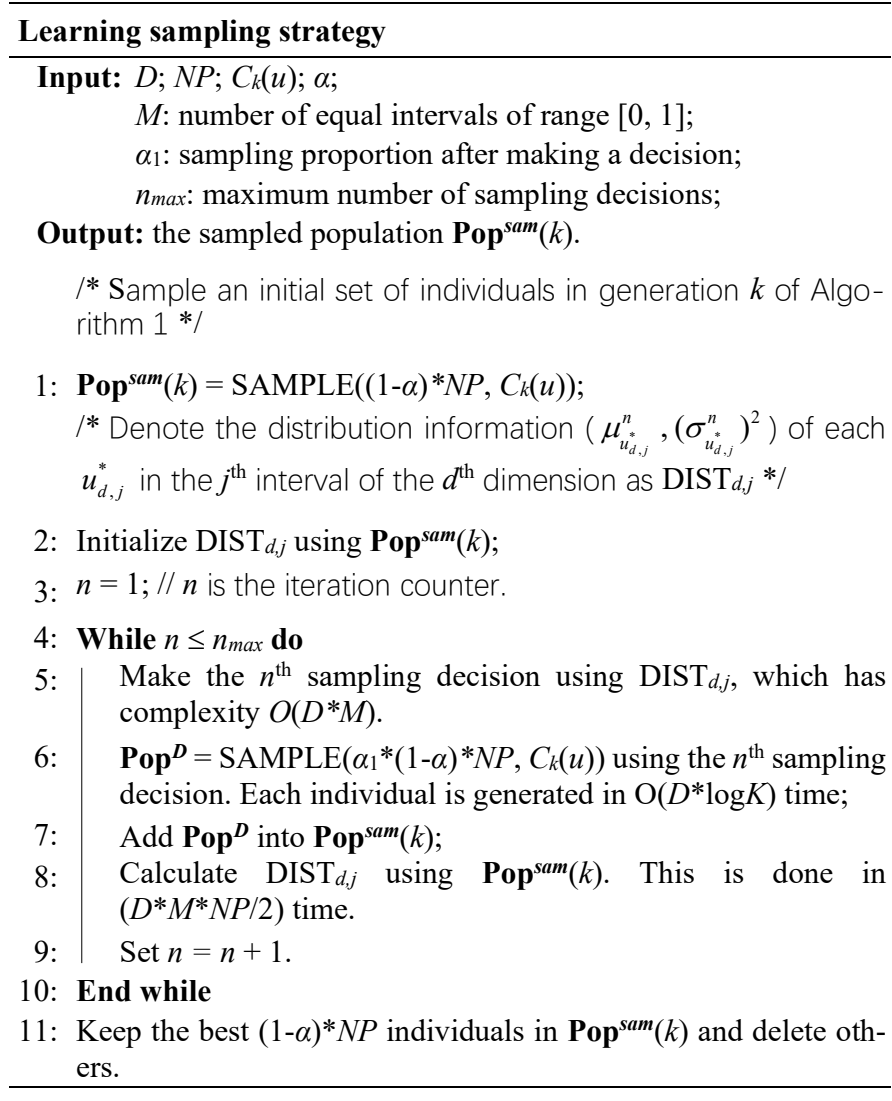

Fig.8. Pseudo-code of learning sampling strategy

Based on the above discussions, a learning sampling strategy is proposed to search for better individuals rapidly. Similar to statistical learning, this learning updates the distribution information of the objective value after taking a sample. It then uses the distribution information to select the best region to sample next. The concept of knowledge gradient introduced in [48] can be applied to mathematically identify which alternative should be sampled next. This is done by determining the region where sample is likely to improve the current best outcome. If the prior distributions of the objective value in the independent alternatives have the same variance and sample error but different means, then the alternative with the best mean should be chosen to sample. But the choice should also depend on the variance and the alternatives with larger variance could be attractive choices as sampling there could get a better objective value. The knowledge gradient gets its name because in a sense it points in the direction of maximal information by taking an expectation of the difference between the current mean and the mean after choosing to sample a certain alternative.

So the task of the learning sampling is to make a decision, which is to select an interval from $M$ distinct intervals for each $u_{d}{ }^{*}, d=1, \ldots, D$. The learning sampling strategy can be outlined as Fig. 8, where $\alpha_{1}$ is the sampling proportion after making a sampling decision; $n_{\max }$ is the maximum number of sampling decisions. The details are described as follows.

The first step of the learning sampling is to sample an initial set of individuals and calculate the prior distribution of objec-

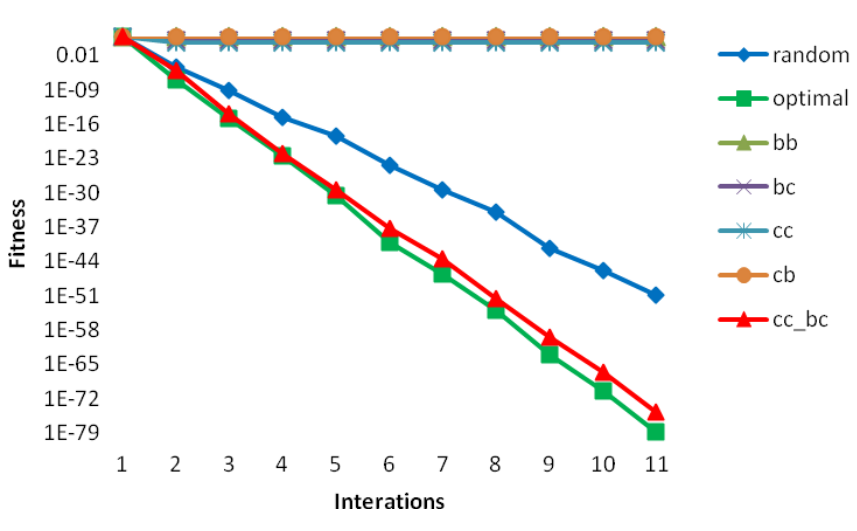

Fig.9. The process of iterations in the different regions on $x_{1}^{2}+x_{2}^{2}$

tive function value according to the statistical information of the individuals located in the $j^{\text {th }}$ interval of the $d^{\text {th }}$ dimension. We assume the prior distribution is a normal distribution with mean $\mu_{u_{d, j}^{*}}^{0}$ and variance $\left(\sigma_{u_{d, j}^{*}}^{0}\right)^{2}$ for each alternative $u_{d, j}^{*}, j=$ $1, \ldots, M$. In the Bayesian view we define $\beta_{u_{d, j}^{*}}^{0}$ as the precision of our belief, which is the inverse of the variance in our estimate $\mu_{u_{d, j}^{*}}^{0}$ of the true mean.

Starting from this initial information, we make a sequence of subsequent sampling decisions, $\left(u_{d, j}^{*}\right)^{1},\left(u_{d, j}^{*}\right)^{2}, \ldots$. Consider the sampling decision $\left(u_{d, j}^{*}\right)^{n}, n=1,2, \ldots$ Assume the sampling error of measuring alternative $u_{d, j}^{*}$ is $\varepsilon_{u_{d, j}^{*}}^{n} \square N\left(0,1 / \beta_{u_{d, j}^{*}}^{\varepsilon}\right)$, where $\beta_{u_{d, j}^{*}}^{\varepsilon}$ is precision of sample, and the resulting sample observation is

$$
\hat{y}_{u_{d, j}^{*}}^{n}=\mu_{u_{d, j}^{*}}^{n-1}+\varepsilon_{u_{d, j}^{*}}^{n}
$$

Because decisions are made sequentially, so the mean and precision can be updated by $\left(u_{d, j}^{*}\right)^{1}, \hat{y}_{u_{d, j}^{*}}^{1}, \ldots,\left(u_{d, j}^{*}\right)^{n}, \hat{y}_{u_{d, j}^{*}}^{n}$. The updating formula is

$$
\begin{aligned}
& \mu_{u_{d, j}^{*}}^{n}=\frac{\beta_{u_{d, j}^{*}}^{n-1} \mu_{u_{d, j}^{n}}^{n-1}+\beta_{u_{d, j}^{*}}^{\varepsilon} \hat{y}_{u_{d, j}^{*}}^{n}}{\beta_{u_{d, j}^{*}}^{n-1}+\beta_{u_{d, j}^{*}}^{\varepsilon}} \\
& \beta_{u_{d, j}^{*}}^{n}=\beta_{u_{d, j}^{*}}^{n-1}+\beta_{u_{d, j}^{*}}^{\varepsilon}
\end{aligned}
$$

The state of knowledge before the $n^{\text {th }}$ sampling is defined as

$$
S_{d, j}^{n-1}=\left(\mu_{u_{d, j}^{*}}^{n-1}, \beta_{u_{d, j}^{*}}^{n-1}\right), j=1, \ldots, M
$$

If we stop measuring now, we would pick the mean of best interval:

$$
V\left(S_{d, j}^{n-1}\right)=\max _{u_{d, j}^{*}} \mu_{u_{d, j}^{*}}^{n-1}, j=1, \ldots, M
$$


Now if we choose measuring alternative $u_{d, j}^{*}$ as the decision, $\left(u_{d, j}^{*}\right)^{n}$, the next state will be $S_{d, j}^{n}=S\left(S^{n-1},\left(u_{d, j}^{*}\right)^{n-1}, \hat{y}_{u_{d, j}^{n}}^{n}\right)$. The best interval would be identified by

$$
V\left(S_{d, j}^{n}\right)=\max _{u_{d, j}^{*}} \mu_{u_{d, j}^{*}}^{n}, j=1, \ldots, M
$$

We would like to choose $u_{d, j}^{*}$ to maximize the expected value of (18). We can also think of (18) as choosing $u_{d, j}^{*}$ to maximize the expected incremental value

$$
v_{u_{d, j}^{*}}^{K G, n}=E\left[V\left(S_{d, j}^{n}\right)\right]-V\left(S_{d, j}^{n-1}\right)
$$

Equation (19) is viewed as the Knowledge Gradient (KG).

The interval to be sampled at the $n^{\text {th }}$ sampling for each variable $j$ can be determined by

$$
\left(u_{d, j}^{*}\right)^{n}=\arg \max _{j} v_{u_{d, j}^{K G, n}}^{K G,}
$$

In our learning sampling strategy, we start by computing the change in the variance of our estimate of $\mu_{u_{d, j}^{*}}^{n}$ given our state of knowledge $S_{d, j}^{n}$.

$$
\begin{aligned}
\left(\tilde{\sigma}_{u_{d, j}^{*}}^{n}\right)^{2} & =\operatorname{Var}\left[\mu_{u_{d, j}^{n}}^{n}-\mu_{u_{d, j}}^{n-1}\right] \\
& =\left(\sigma_{u_{d, j}^{*}}^{n-1}\right)^{2}-\left(\sigma_{u_{d, j}^{*}}^{n}\right)^{2} \\
& =\frac{\left(\sigma_{u_{d, j}^{*}}^{n-1}\right)^{2}}{1+\left(1 / \beta_{u_{d, j}^{*}}^{\varepsilon}\right)^{2} /\left(\sigma_{u_{d, j}^{*}}^{n-1}\right)^{2}}
\end{aligned}
$$

We then compute normalized influence of decision $\left(u_{d, j}^{*}\right)^{n}$, calculated by current estimate of the value of $\mu_{u_{d, j}^{*}}^{n}$, and the best of the rest, and the number of standard deviations, given by

$$
\zeta_{u_{d, j}^{*}}^{n}=-\left|\left(\mu_{u_{d, j}^{n}}^{n-1}-\max _{u_{d, j}^{*} \neq \neq u_{d, j}^{*}} \mu_{u_{d, j}^{n}}^{n-1}\right) / \tilde{\sigma}_{u_{d, j}^{*}}^{n}\right|
$$

Next, we use a standard formula given by

$$
f(\zeta)=\zeta \Phi(\zeta)+\varphi(\zeta)
$$

where $\Phi(\zeta)$ and $\varphi(\zeta)$ are the cumulative probability function and the density function, respectively, of the standard normal distribution. Finally, the Knowledge Gradient is given by

$$
v_{u_{d, j}^{K}}^{K G, n}=\left(\tilde{\sigma}_{u_{d, j}^{*}}^{n}\right)^{2} f\left(\zeta_{u_{d, j}^{*}}^{n}\right)
$$

Then we can make the sampling decision according to (20).

As Fig. 9 shows, the solution obtained by learning sampling strategy is better than other methods with the same number of iterations.

For the complexity, we remark that the learning sampling strategy has $O\left(n_{\max } D M N P / 2\right)+O(D \log K(1-\alpha) N P)+O\left(n_{\max } D\right.$ $M$ ) steps. Note that $M, K$ and $n_{\max }$ are small constants. So the time complexity is $O(c D N P)$, here $c$ is a small positive integer.

\section{EXPERIMENTAL STUDIES}

In order to test the performance of the proposed algorithm, a comprehensive series of benchmark functions of 10/30/50/ 100/500 dimensions are used. The algorithms are developed using C\# language on the platform of Microsoft Visual Studio 2005. All experiments are carried out on a Lenovo personal computer with 3.30GHz Intel CPU and 4GB RAM.

\section{A. Test Problems}

1) CEC 2013 benchmark suite: The 28 benchmark functions in the CEC 2013 benchmark suite are utilized to evaluate the algorithm performance, among which $F_{1}$ to $F_{5}$ are unimodal functions; $F_{6}$ to $F_{20}$ are basic multimodal functions; $F_{21}$ to $F_{28}$ are composition functions. The problem definitions and evaluation criteria can be obtained from literature [49].

2) The scaling-up test functions: To further test the performance and the universality of our proposed algorithm, the 13 classical functions are selected from [12] and used. They are presented in TABLE I, which can be found in the supplementary file of the paper. These are minimization problems and can be classified into three groups as follows. $F_{29}$ and $F_{30}$ are separable unimodal problems; $F_{31}$ to $F_{37}$ belong to non-separable problems with only a few local optima; $F_{38}$ to $F_{41}$ are multimodal problems with many local optima. $F_{30}, F_{32}, F_{34}, F_{36}$, and $F_{39}$ are the shifted or rotated function of $F_{29}, F_{31}, F_{33}, F_{35}$, and $F_{38}$, respectively, and the values of the shifted global optima and the transformation matrices can be obtained in [50]. More details about these functions can be found in $[50,51]$.

\section{B. Comparison Strategies and Metrics}

For each algorithm in this paper, we use $x^{*}$ and $x^{\prime}$ to indicate the known optimum solution and the best solution obtained when the stopping criterion is satisfied, respectively. Therefore, the function error value $\left(F\left(x^{\prime}\right)-F\left(x^{*}\right)\right)$ is employed to evaluate the algorithms' performance, and if the value is below 1e-8, we see it as zero. The mean and variance are calculated through many independent runs for CEC 2013 benchmark functions and the scaling-up test functions. In each run the algorithms are executed for dimension $\times 10000$ Fitness Evaluations [50] and dimension $\times 11000$ Fitness Evaluations [12] on the two classes of problems respectively. This setting is the same for all algorithms tested. The mean error and the corresponding standard deviation are calculated and presented in the numerical results tables, where the best mean error value is shown in bold for each benchmark function. The standard deviation appears after the " \pm " sign. Because of the space limitation, some tables are placed in the supplementary file of the paper. 
TABLE II

PARAMETERS OF THE THE PROPOSED ALGORITHM

\begin{tabular}{cc}
\hline \hline Parameter & Value \\
\hline$N P$ & 400 \\
$\alpha$ & 0.6 \\
$p_{0}$ & 0.1 \\
$K$ & 2 \\
$M$ & 2 \\
$\alpha_{1}$ & 0.1 \\
$n_{\max }$ & 1 \\
$\beta_{u_{d, j}^{*}}^{\varepsilon}$ & 0.1 \\
\hline \hline
\end{tabular}

To get a direct indication of the performance of our proposed algorithm, the number of the best results in each test group are listed at the bottom of the numerical results tables.

To obtain a statistical conclusion, the two-sided Wilcoxon rank sum test [52] is employed to evaluate the performance between EDA-FL and other algorithms. The value of significance level is set to be 0.05 . The marks "+", "-" and "=" indicate that the compared algorithm is superior to EDA-FL, EDA-FL performs better, and there is no statistically significant difference between EDA-FL and compared algorithm, respectively.

Moreover, to evaluate the performance of the convergence, the convergence curves about the mean error values of the best solutions for each algorithm are presented.

To have a reliable and fair comparison, the experimental results of all competitor algorithms are the reported data from the original papers or the websites of authors.

\section{Parameter Analytics}

In our proposed algorithm with filtering framework only (EDA-F), the step of prediction has great influence, where $\boldsymbol{\Lambda}$ is a diagonal matrix with $D \times D$ dimension. The diagonal elements represent the noise level. To increase the randomness of noise, we set $p_{0}$ is 0.1 . The constant values of the diagonal elements are set as 0.9. Similarly, the step of revision plays an important role in our algorithm. The performance has great differences for different combinations of $p_{1}$ and $p_{2}$ values. To obtain the best combination, we test some representative functions with different $p_{1}$ and $p_{2}$. The other parameters for the algorithm are given in TABLE II. The means and standard deviations for 10 runs are listed into the TABLE III. As TABLE III shows, when the best prediction individual is revised through formula (12), the algorithm achieves the best solutions in 14 cases for $10 / 30 / 50$ dimension. Regarding the total number of the best solutions obtained by the algorithm with other parameters settings, $\left(p_{1}=0, p_{2}=0\right),\left(p_{1}=0.05, p_{2}=0.05\right),\left(p_{1}=0.1, p_{2}=0\right)$ and $\left(p_{1}=0.2, p_{2}=0.4\right)$, are $9,10,7$ and 6 , respectively.

Result analysis - When $p_{1}=0$ and $p_{2}=0$ indicating that there are no revised individuals going into modeling individuals set, the performance is not good. This proves that the operation "Revise" in filtering is effective to improve the performance. When the values of $p_{1}$ and $p_{2}$ increase to large values, the performance shows a decreasing trend. The root cause of this is that the revised individuals can increase the diversity of the

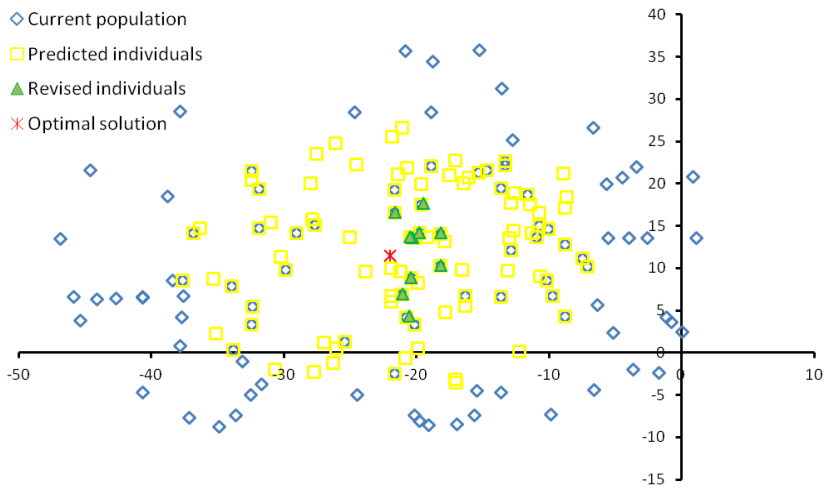

(a) $\boldsymbol{F}_{2}$
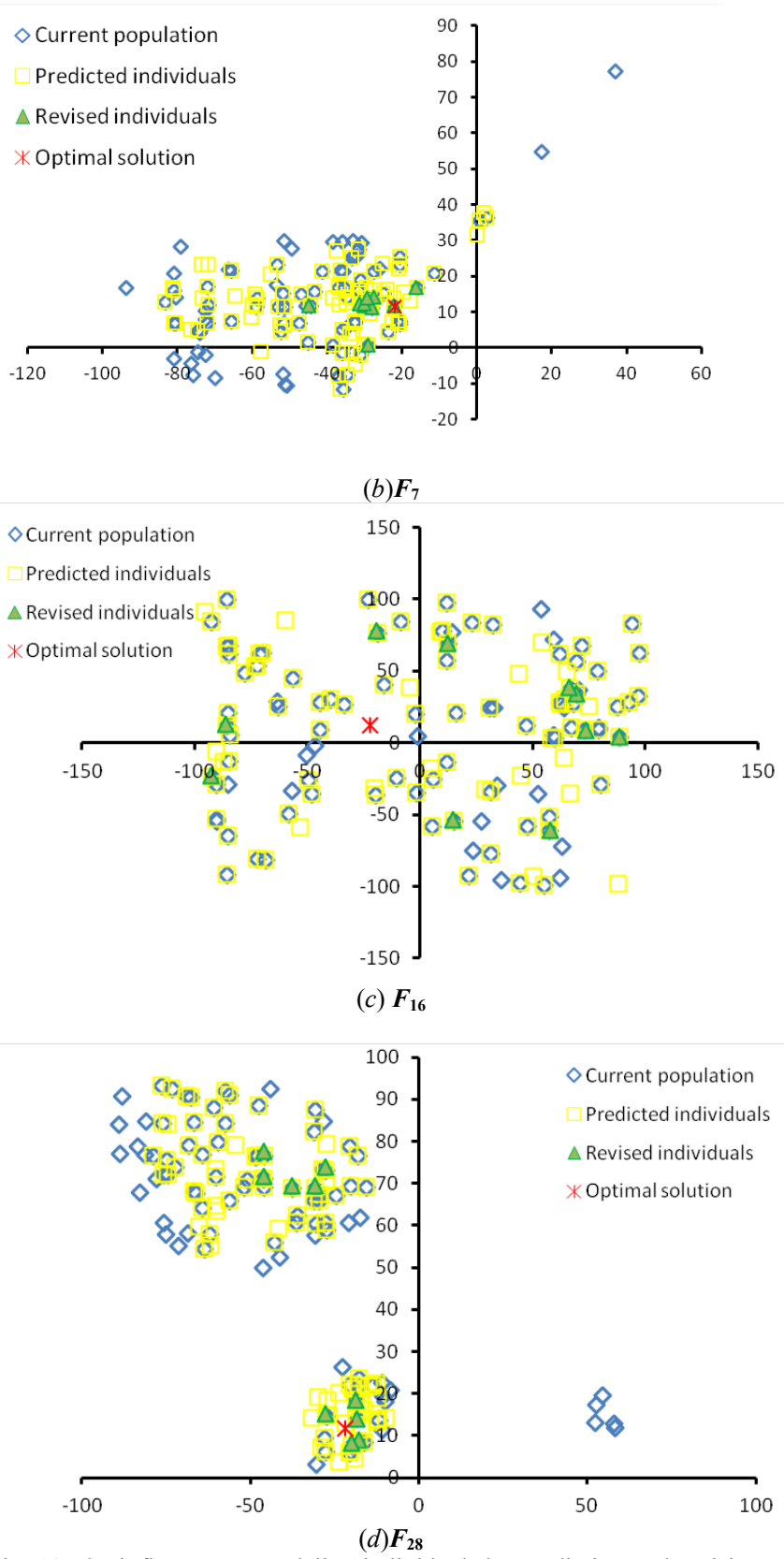

Fig. 10 The influence on modeling individuals by predicting and revising on the function $F_{2}, F_{7}, F_{16}$ and $F_{28}$ 
modeling population. When the individuals are revised too much, the precision of distribution will decrease.

TABLE III

MEAN AND STANDARD DEVIATIONS OF BENCHMARK TEST FUNCTIONS FOR PARAMETER ANALYSIS

\begin{tabular}{|c|c|c|c|c|c|c|}
\hline Pro. & $D$ & $\begin{array}{c}\text { EDA-F } \\
\left(p_{1}=0, p_{2}=0\right) \\
\text { Mean } / \text { Std }\end{array}$ & $\begin{array}{c}\text { EDA-F } \\
\text { (best prediction) } \\
\text { Mean/Std }\end{array}$ & $\begin{array}{c}\text { EDA-F } \\
\left(p_{1}=0.05, p_{2}=0.05\right) \\
\text { Mean } / \text { Std }\end{array}$ & $\begin{array}{c}\text { EDA-F } \\
\left(p_{1}=0.1, p_{2}=0\right) \\
\text { Mean } / \text { Std }\end{array}$ & $\begin{array}{c}\text { EDA-F } \\
\left(p_{1}=0.2, p_{2}=0.4\right) \\
\text { Mean } / \text { Std }\end{array}$ \\
\hline \multirow{3}{*}{$F_{1}$} & 10 & $0.00 \mathrm{e}+00 \pm 0.00 \mathrm{e}+00$ & $0.00 \mathrm{e}+00 \pm 0.00 \mathrm{e}+00$ & $0.00 \mathrm{e}+00 \pm 0.00 \mathrm{e}+00$ & $0.00 \mathrm{e}+00 \pm 0.00 \mathrm{e}+00$ & $0.00 \mathrm{e}+00 \pm 0.00 \mathrm{e}+00$ \\
\hline & 30 & $0.00 \mathrm{e}+00 \pm 0.00 \mathrm{e}+00$ & $0.00 \mathrm{e}+00 \pm 0.00 \mathrm{e}+00$ & $0.00 \mathrm{e}+00 \pm 0.00 \mathrm{e}+00$ & $0.00 \mathrm{e}+00 \pm 0.00 \mathrm{e}+00$ & $0.00 \mathrm{e}+00 \pm 0.00 \mathrm{e}+00$ \\
\hline & 50 & $0.00 \mathrm{e}+00 \pm 0.00 \mathrm{e}+00$ & $0.00 \mathrm{e}+00 \pm 0.00 \mathrm{e}+00$ & $0.00 \mathrm{e}+00 \pm 0.00 \mathrm{e}+00$ & $0.00 \mathrm{e}+00 \pm 0.00 \mathrm{e}+00$ & $0.00 \mathrm{e}+00 \pm 0.00 \mathrm{e}+00$ \\
\hline \multirow{3}{*}{$F_{2}$} & 10 & $1.14 \mathrm{e}-01 \pm 1.63 \mathrm{e}-01$ & $3.96 \mathrm{e}-02 \pm 3.70 \mathrm{e}-02$ & $8.88 \mathrm{e}-05 \pm 1.12 \mathrm{e}-04$ & $3.61 e-05 \pm 5.93 e-05$ & $3.53 \mathrm{e}+00 \pm 9.20 \mathrm{e}+00$ \\
\hline & 30 & $4.14 \mathrm{e}+00 \pm 2.18 \mathrm{e}+00$ & $1.47 \mathrm{e}+00 \pm 1.32 \mathrm{e}+00$ & $7.72 \mathrm{e}+00 \pm 3.25 \mathrm{e}+01$ & $1.50 \mathrm{e}+00 \pm 3.06 \mathrm{e}+00$ & $1.46 \mathrm{e}+01 \pm 3.26 \mathrm{e}+01$ \\
\hline & 50 & $1.18 \mathrm{e}+01 \pm 5.82 \mathrm{e}+00$ & $9.15 \mathrm{e}+00 \pm 8.50 \mathrm{e}+00$ & $2.76 \mathrm{e}+00 \pm 4.55 \mathrm{e}+00$ & $6.42 \mathrm{e}+00 \pm 8.29 \mathrm{e}+00$ & $7.88 \mathrm{e}+01 \pm 1.10 \mathrm{e}+02$ \\
\hline \multirow{3}{*}{$F_{16}$} & 10 & $1.42 \mathrm{e}+00 \pm 2.49 \mathrm{e}-01$ & $3.47 \mathrm{e}-02 \pm 2.34 \mathrm{e}-02$ & $1.54 \mathrm{e}-01 \pm 1.66 \mathrm{e}-01$ & $1.10 \mathrm{e}-01 \pm 7.80 \mathrm{e}-02$ & $1.95 \mathrm{e}-01 \pm 1.25 \mathrm{e}-01$ \\
\hline & 30 & $2.89 \mathrm{e}+00 \pm 5.98 \mathrm{e}-01$ & $3.81 \mathrm{e}-01 \pm 2.00 \mathrm{e}-01$ & $5.44 \mathrm{e}-01 \pm 3.00 \mathrm{e}-01$ & $5.94 \mathrm{e}-01 \pm 2.89 \mathrm{e}-01$ & $5.78 \mathrm{e}-01 \pm 3.81 \mathrm{e}-01$ \\
\hline & 50 & $3.66 \mathrm{e}+00 \pm 8.42 \mathrm{e}-01$ & $6.80 \mathrm{e}-01 \pm 3.77 \mathrm{e}-01$ & $8.30 \mathrm{e}-01 \pm 3.41 \mathrm{e}-01$ & $9.60 \mathrm{e}-01 \pm 4.39 \mathrm{e}-01$ & $1.02 \mathrm{e}+00 \pm 3.99 \mathrm{e}-01$ \\
\hline \multirow{3}{*}{$F_{17}$} & 10 & $9.29 \mathrm{e}+00 \pm 3.07 \mathrm{e}+00$ & $1.03 \mathrm{e}+01 \pm 1.38 \mathrm{e}-01$ & $9.30 \mathrm{e}+00 \pm 3.08 \mathrm{e}+00$ & $9.79 \mathrm{e}+00 \pm 2.21 \mathrm{e}+00$ & $1.03 \mathrm{e}+01 \pm 2.30 \mathrm{e}+00$ \\
\hline & 30 & $3.12 \mathrm{e}+01 \pm 4.32 \mathrm{e}-01$ & $3.11 \mathrm{e}+01 \pm 3.04 \mathrm{e}-01$ & $3.11 \mathrm{e}+01 \pm 4.13 \mathrm{e}-01$ & $3.12 \mathrm{e}+01 \pm 6.61 \mathrm{e}-01$ & $3.15 \mathrm{e}+01 \pm 4.62 \mathrm{e}-01$ \\
\hline & 50 & $5.23 \mathrm{e}+01 \pm 1.45 \mathrm{e}+00$ & $5.21 \mathrm{e}+01 \pm 1.55 \mathrm{e}+00$ & $5.26 \mathrm{e}+01 \pm 1.47 \mathrm{e}+00$ & $5.29 \mathrm{e}+01 \pm 1.76 \mathrm{e}+00$ & $5.29 \mathrm{e}+01 \pm 7.51 \mathrm{e}-01$ \\
\hline \multirow{3}{*}{$F_{19}$} & 10 & $3.93 \mathrm{e}-01 \pm 1.31 \mathrm{e}-01$ & $4.40 \mathrm{e}-01 \pm 1.31 \mathrm{e}-01$ & $3.66 \mathrm{e}-01 \pm 1.30 \mathrm{e}-01$ & $4.61 \mathrm{e}-01 \pm 1.14 \mathrm{e}-01$ & $4.81 \mathrm{e}-01 \pm 1.75 \mathrm{e}-01$ \\
\hline & 30 & $1.09 \mathrm{e}+00 \pm 2.41 \mathrm{e}-01$ & $1.13 \mathrm{e}+00 \pm 2.65 \mathrm{e}-01$ & $1.17 \mathrm{e}+00 \pm 2.58 \mathrm{e}-01$ & $1.31 \mathrm{e}+00 \pm 2.00 \mathrm{e}-01$ & $1.48 \mathrm{e}+00 \pm 3.27 \mathrm{e}-01$ \\
\hline & 50 & $2.15 \mathrm{e}+00 \pm 4.69 \mathrm{e}-01$ & $1.88 \mathrm{e}+00 \pm 3.68 \mathrm{e}-01$ & $2.20 \mathrm{e}+00 \pm 4.98 \mathrm{e}-01$ & $2.47 \mathrm{e}+00 \pm 5.31 \mathrm{e}-01$ & $2.60 \mathrm{e}+00 \pm 4.76 \mathrm{e}-01$ \\
\hline \multirow{3}{*}{$F_{26}$} & 10 & $1.91 \mathrm{e}+02 \pm 2.74 \mathrm{e}+01$ & $1.96 \mathrm{e}+02 \pm 1.76 \mathrm{e}+01$ & $1.96 \mathrm{e}+02 \pm 1.81 \mathrm{e}+01$ & $1.92 \mathrm{e}+02 \pm 2.47 \mathrm{e}+01$ & $1.96 \mathrm{e}+02 \pm 1.68 \mathrm{e}+01$ \\
\hline & 30 & $2.00 \mathrm{e}+02 \pm 3.10 \mathrm{e}-07$ & $2.00 \mathrm{e}+02 \pm 3.25 \mathrm{e}-07$ & $2.00 \mathrm{e}+02 \pm 5.63 \mathrm{e}-06$ & $2.00 \mathrm{e}+02 \pm 4.57 \mathrm{e}-06$ & $2.00 \mathrm{e}+02 \pm 2.49 \mathrm{e}-06$ \\
\hline & 50 & $2.00 e+02 \pm 1.78 e-06$ & $2.00 \mathrm{e}+02 \pm 1.30 \mathrm{e}-06$ & $2.00 \mathrm{e}+02 \pm 7.04 \mathrm{e}-06$ & $2.00 e+02 \pm 1.30 e-05$ & $2.00 e+02 \pm 1.56 e-05$ \\
\hline \multirow{3}{*}{$F_{28}$} & 10 & $2.70 \mathrm{e}+02 \pm 7.33 \mathrm{e}+01$ & $2.68 \mathrm{e}+02 \pm 7.48 \mathrm{e}+01$ & $2.80 \mathrm{e}+02 \pm 6.16 \mathrm{e}+01$ & $2.90 \mathrm{e}+02 \pm 4.47 \mathrm{e}+01$ & $2.80 \mathrm{e}+02 \pm 6.16 \mathrm{e}+01$ \\
\hline & 30 & $3.00 \mathrm{e}+02 \pm 5.29 \mathrm{e}-13$ & $3.00 \mathrm{e}+02 \pm 5.68 \mathrm{e}-13$ & $3.00 \mathrm{e}+02 \pm 4.42 \mathrm{e}-08$ & $3.00 \mathrm{e}+02 \pm 2.19 \mathrm{e}-08$ & $3.00 \mathrm{e}+02 \pm 6.17 \mathrm{e}-03$ \\
\hline & 50 & $5.50 \mathrm{e}+02 \pm 6.71 \mathrm{e}+02$ & $5.48 \mathrm{e}+02 \pm 6.64 \mathrm{e}+02$ & $4.00 \mathrm{e}+02 \pm 7.47 \mathrm{e}-11$ & $5.52 \mathrm{e}+02 \pm 6.81 \mathrm{e}+02$ & $5.55 \mathrm{e}+02 \pm 6.92 \mathrm{e}+02$ \\
\hline \multicolumn{2}{|c|}{ No of Best } & 9 & 14 & 10 & 7 & 6 \\
\hline
\end{tabular}

TABLE IV

MEAN AND STANDARD DEVIATIONS OF RESULTS ON 10-D BENCHMARK TEST FUNCTIONS

\begin{tabular}{|c|c|c|c|}
\hline $10-\mathrm{D}$ & $\begin{array}{c}\text { EDA } \\
\text { Mean/Std }\end{array}$ & $\begin{array}{c}\text { EDA-F } \\
\text { Mean/Std }\end{array}$ & $\begin{array}{l}\text { EDA-FL } \\
\text { Mean/Std }\end{array}$ \\
\hline$F_{1}$ & $5.21 \mathrm{e}-01 \pm 8.12 \mathrm{e}-01$ & $0.00 \mathrm{e}+00 \pm 0.00 \mathrm{e}+00$ & $0.00 \mathrm{e}+00 \pm 0.00 \mathrm{e}+00$ \\
\hline$F_{2}$ & $8.03 \mathrm{e}+03 \pm 2.72 \mathrm{e}+03$ & $3.96 \mathrm{e}-02 \pm 3.70 \mathrm{e}-02$ & $3.87 \mathrm{e}-04 \pm 2.82 \mathrm{e}-04$ \\
\hline$F_{3}$ & $1.27 \mathrm{e}+08 \pm 1.65 \mathrm{e}+08$ & $1.17 \mathrm{e}+01 \pm 1.35 \mathrm{e}+01$ & $1.26 \mathrm{e}+00 \pm 2.31 \mathrm{e}+00$ \\
\hline$F_{4}$ & $7.74 \mathrm{e}+03 \pm 2.92 \mathrm{e}+03$ & $2.51 \mathrm{e}-02 \pm 1.90 \mathrm{e}-02$ & $3.24 \mathrm{e}-04 \pm 2.47 \mathrm{e}-04$ \\
\hline$F_{5}$ & $2.22 \mathrm{e}+00 \pm 3.93 \mathrm{e}+00$ & $0.00 \mathrm{e}+00 \pm 0.00 \mathrm{e}+00$ & $0.00 \mathrm{e}+00 \pm 0.00 \mathrm{e}+00$ \\
\hline$F_{6}$ & $1.84 \mathrm{e}+01 \pm 7.93 \mathrm{e}+00$ & $4.71 \mathrm{e}+00 \pm 5.00 \mathrm{e}+00$ & $4.32 \mathrm{e}+00 \pm 4.97 \mathrm{e}+00$ \\
\hline$F_{7}$ & $1.28 \mathrm{e}+01 \pm 5.74 \mathrm{e}+00$ & $4.90 \mathrm{e}-02 \pm 1.88 \mathrm{e}-01$ & $1.78 \mathrm{e}-03 \pm 8.74 \mathrm{e}-03$ \\
\hline$F_{8}$ & $2.05 \mathrm{e}+01 \pm 0.00 \mathrm{e}+00$ & $2.03 e+01 \pm 0.00 e+00$ & $2.03 e+01 \pm 0.00 e+00$ \\
\hline$F_{9}$ & $3.05 \mathrm{e}+00 \pm 1.06 \mathrm{e}+00$ & $1.87 \mathrm{e}+00 \pm 1.28 \mathrm{e}+00$ & $1.91 \mathrm{e}+00 \pm 1.54 \mathrm{e}+00$ \\
\hline$F_{10}$ & $6.05 \mathrm{e}+00 \pm 4.86 \mathrm{e}+00$ & $2.24 \mathrm{e}-01 \pm 1.35 \mathrm{e}-01$ & $9.39 \mathrm{e}-02 \pm 7.56 \mathrm{e}-02$ \\
\hline$F_{11}$ & $7.29 \mathrm{e}-01 \pm 7.64 \mathrm{e}-01$ & $0.00 \mathrm{e}+00 \pm 0.00 \mathrm{e}+00$ & $0.00 \mathrm{e}+00 \pm 0.00 \mathrm{e}+00$ \\
\hline$F_{12}$ & $1.05 \mathrm{e}+01 \pm 6.54 \mathrm{e}+00$ & $1.08 \mathrm{e}+01 \pm 6.02 \mathrm{e}+00$ & $1.08 \mathrm{e}+01 \pm 4.42 \mathrm{e}+00$ \\
\hline$F_{13}$ & $1.35 \mathrm{e}+01 \pm 7.56 \mathrm{e}+00$ & $1.66 \mathrm{e}+01 \pm 7.64 \mathrm{e}+00$ & $1.47 \mathrm{e}+01 \pm 7.56 \mathrm{e}+00$ \\
\hline$F_{14}$ & $2.73 \mathrm{e}+00 \pm 3.05 \mathrm{e}+00$ & $8.40 \mathrm{e}-01 \pm 1.41 \mathrm{e}+00$ & $1.43 \mathrm{e}+00 \pm 1.88 \mathrm{e}+00$ \\
\hline$F_{15}$ & $1.23 \mathrm{e}+03 \pm 1.64 \mathrm{e}+02$ & $8.48 \mathrm{e}+02 \pm 2.68 \mathrm{e}+02$ & $9.28 \mathrm{e}+02 \pm 3.06 \mathrm{e}+02$ \\
\hline$F_{16}$ & $1.14 \mathrm{e}+00 \pm 1.83 \mathrm{e}-01$ & $3.47 \mathrm{e}-02 \pm 2.34 \mathrm{e}-02$ & $1.42 \mathrm{e}-02 \pm 1.36 \mathrm{e}-02$ \\
\hline$F_{17}$ & $1.11 \mathrm{e}+01 \pm 1.65 \mathrm{e}+00$ & $1.03 \mathrm{e}+01 \pm 2.40 \mathrm{e}-01$ & $9.31 \mathrm{e}+00 \pm 3.31 \mathrm{e}+00$ \\
\hline$F_{18}$ & $2.96 \mathrm{e}+01 \pm 3.85 \mathrm{e}+00$ & $2.07 \mathrm{e}+01 \pm 4.87 \mathrm{e}+00$ & $1.95 e+01 \pm 5.56 e+00$ \\
\hline$F_{19}$ & $5.83 \mathrm{e}-01 \pm 1.96 \mathrm{e}-01$ & $4.40 \mathrm{e}-01 \pm 1.31 \mathrm{e}-01$ & $5.12 \mathrm{e}-01 \pm 1.77 \mathrm{e}-01$ \\
\hline$F_{20}$ & $2.89 \mathrm{e}+00 \pm 4.80 \mathrm{e}-01$ & $2.81 \mathrm{e}+00 \pm 6.01 \mathrm{e}-01$ & $2.56 \mathrm{e}+00 \pm 4.88 \mathrm{e}-01$ \\
\hline$F_{21}$ & $4.00 \mathrm{e}+02 \pm 3.73 \mathrm{e}-01$ & $4.00 \mathrm{e}+02 \pm 2.28 \mathrm{e}-13$ & $3.92 \mathrm{e}+02 \pm 4.00 \mathrm{e}+01$ \\
\hline$F_{22}$ & $3.68 \mathrm{e}+01 \pm 3.30 \mathrm{e}+01$ & $4.52 \mathrm{e}+01 \pm 5.44 \mathrm{e}+01$ & $4.09 \mathrm{e}+01 \pm 4.99 \mathrm{e}+01$ \\
\hline$F_{23}$ & $9.81 \mathrm{e}+02 \pm 2.65 \mathrm{e}+02$ & $9.41 \mathrm{e}+02 \pm 2.82 \mathrm{e}+02$ & $9.18 \mathrm{e}+02 \pm 3.36 \mathrm{e}+02$ \\
\hline$F_{24}$ & $2.11 \mathrm{e}+02 \pm 3.50 \mathrm{e}+00$ & $2.02 \mathrm{e}+02 \pm 3.65 \mathrm{e}+00$ & $2.01 \mathrm{e}+02 \pm 3.42 \mathrm{e}+00$ \\
\hline$F_{25}$ & $2.08 \mathrm{e}+02 \pm 4.43 \mathrm{e}+00$ & $1.93 \mathrm{e}+02 \pm 2.44 \mathrm{e}+01$ & $1.94 \mathrm{e}+02 \pm 2.23 \mathrm{e}+01$ \\
\hline$F_{26}$ & $1.91 \mathrm{e}+02 \pm 2.70 \mathrm{e}+01$ & $1.96 \mathrm{e}+02 \pm 1.76 \mathrm{e}+01$ & $1.93 \mathrm{e}+02 \pm \quad 2.35 \mathrm{e}+01$ \\
\hline$F_{27}$ & $3.55 \mathrm{e}+02 \pm 3.46 \mathrm{e}+01$ & $3.00 \mathrm{e}+02 \pm 4.83 \mathrm{e}-03$ & $3.00 \mathrm{e}+02 \pm 9.53 \mathrm{e}-05$ \\
\hline$F_{28}$ & $3.23 \mathrm{e}+02 \pm 2.41 \mathrm{e}+01$ & $2.68 \mathrm{e}+02 \pm 7.48 \mathrm{e}+01$ & $2.92 \mathrm{e}+02 \pm 4.00 \mathrm{e}+01$ \\
\hline No of Best & 4 & 11 & 19 \\
\hline
\end{tabular}




\section{Experimental Results}

\section{1) Experiment series 1 (Evaluation on the strategies):}

In our proposed algorithm, there are two innovation points: a new framework with filtering and a strategy of learning sampling. To evaluate the performance of them, three versions of the algorithm, EDA, EDA-F and EDA-FL, are tested on CEC 2013 benchmark functions with 10-D. The parameters are set as in TABLE II. The means and standard deviations for 10 runs are presented in TABLE IV. The best result (with the minimal mean value) is bolded in each row. The results indicate that EDA-F is better than EDA and EDA-FL is superior to EDA-F according to the number of best solutions. To further demonstrate the performance and mechanism of filtering, a class of experiments is executed to display the locations of current population, predicted individuals, revised individuals and optimal solution in certain iteration. Fig. 10 shows the influence on modeling individuals by predicting and revising on 2- $D$ function $F_{2}, F_{7}, F_{16}$ and $F_{28}$. The red asterisk denotes the optimal solution. The yellow square box and green triangle indicate the predicted individuals and revised individuals, which tend to be closer to the optimal solution. The convergence sketches on 10-D function $F_{2}, F_{7}, F_{16}$ and $F_{28}$ are presented in Fig. 11, where we can see that EDA-FL has a quick convergence speed.

Result analysis - In our new EDA, the potential advantage of the two distinct operations "Predict" and "Revise" is illustrated in Fig.10. The predicted and revised individuals not only tend to be closer to the optimal solution but also increase the diversity of the population and so make it evolve further. Moreover, when adding the learning sampling strategy, our algorithm converges faster. This point is consistent with the idea of getting a good sample through learning.

2) Experiment series 2 (comparison of EDA-FL with other state-of-the-art Evolutionary algorithms for CEC 2013 benchmark suite with 10/30/50 dimension):

To embody the competitiveness of our proposed algorithm, in this subsection, we compare the proposed EDA-FL with three $\mathrm{DE}$ algorithms that have outstanding performance, among the state-of-the-art intelligent algorithms, including CoDE [53], JADE [54], and SaDE [55]. The parameters are set as in TABLE II. The means and standard deviations for 10 runs are presented in TABLE V, VI and VII, which can be found in the supplementary file of the paper. The results of CoDE, JADE and $\mathrm{SaDE}$ are from [52]. Fig. 12 shows that the number of cases on which each algorithm performs the best in the comparison between EDA-FL and state-of-the art DE algorithms. To show the performance more clearly, the statistical significance results are summarized in Fig. 13.

Result analysis - With great exploitation ability, our proposed algorithm has great advantages on the unimodal function $F_{1}-F_{5}$. Besides, when the dimension increases to 50 , the algorithm outperforms all other three algorithms, showing that our proposed algorithm EDA-FL has strong competitiveness.

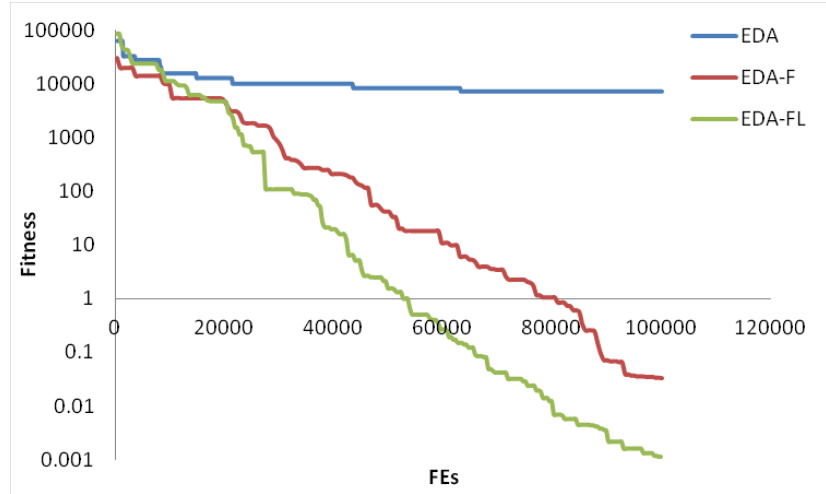

(a) $F_{2}$

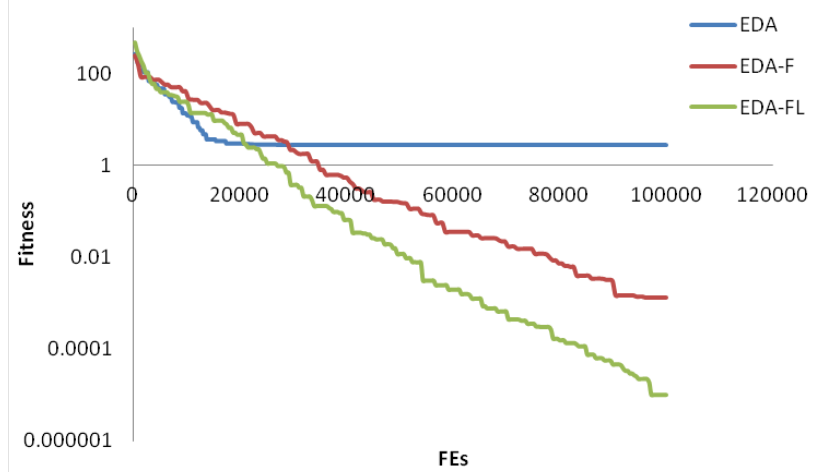

(b) $F_{7}$

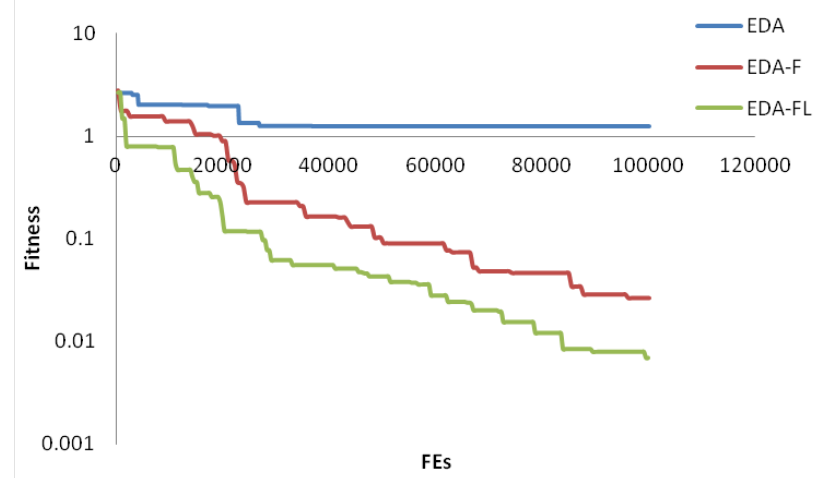

(c) $F_{16}$

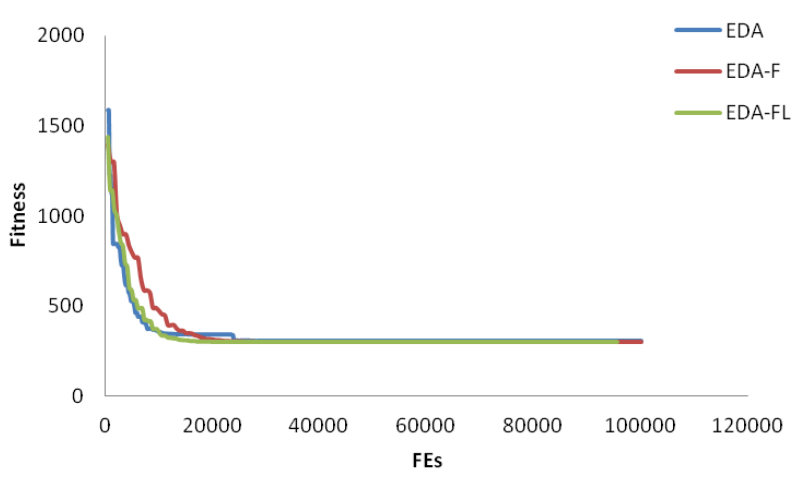

(d) $F_{28}$

Fig. 11 The convergence sketches of the function $F_{2}, F_{7}, F_{16}$ and $F_{28}$ 


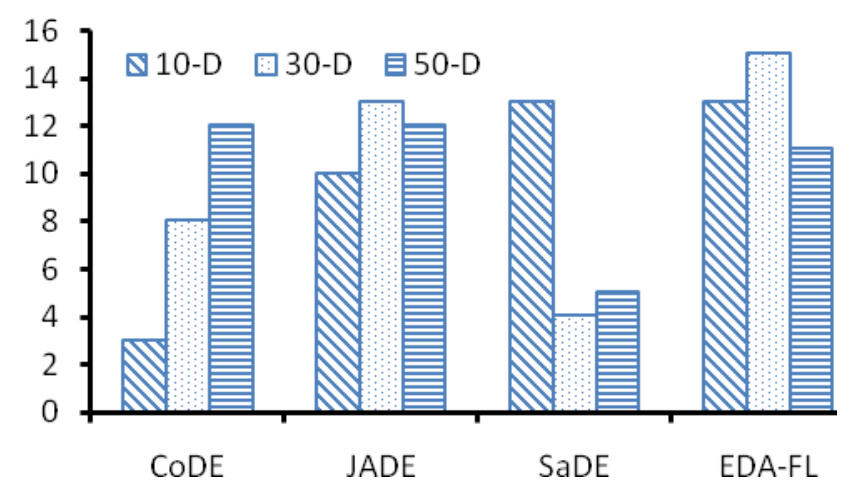

Fig. 12 The number of cases on which each algorithm performs the best in the comparison between EDA-FL and state-of-the-art DE algorithms.

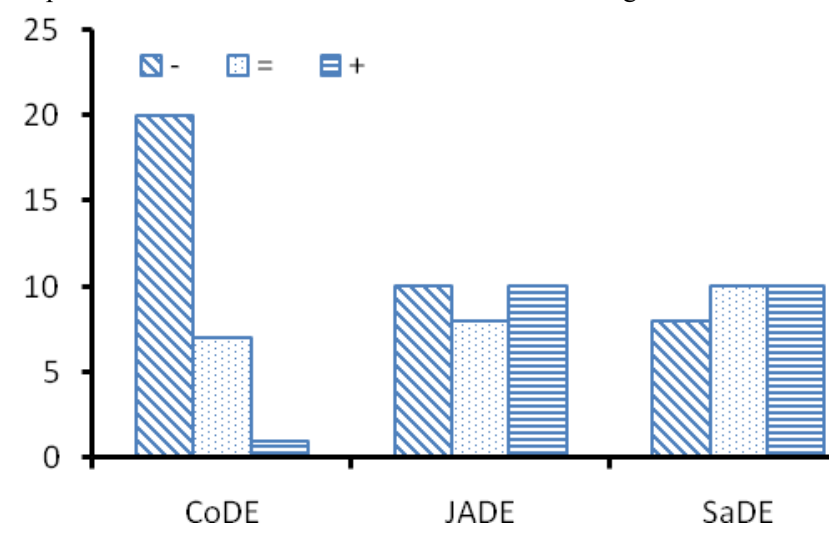

(a)

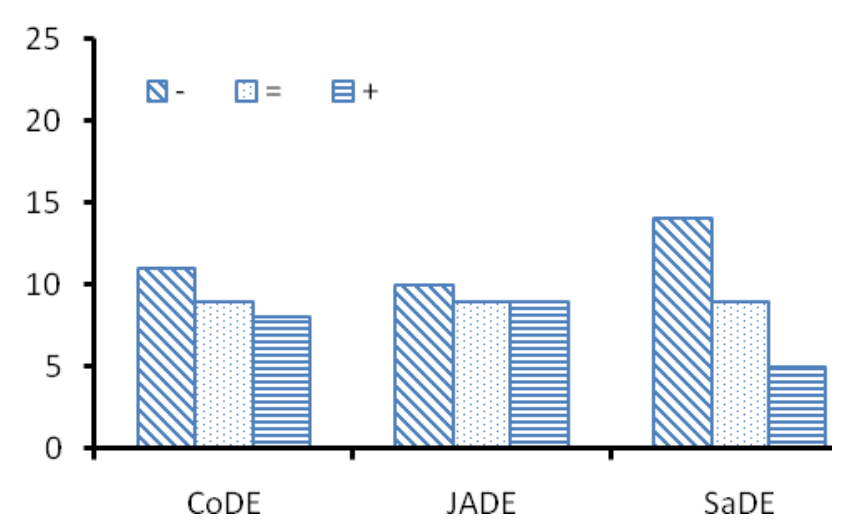

(b)

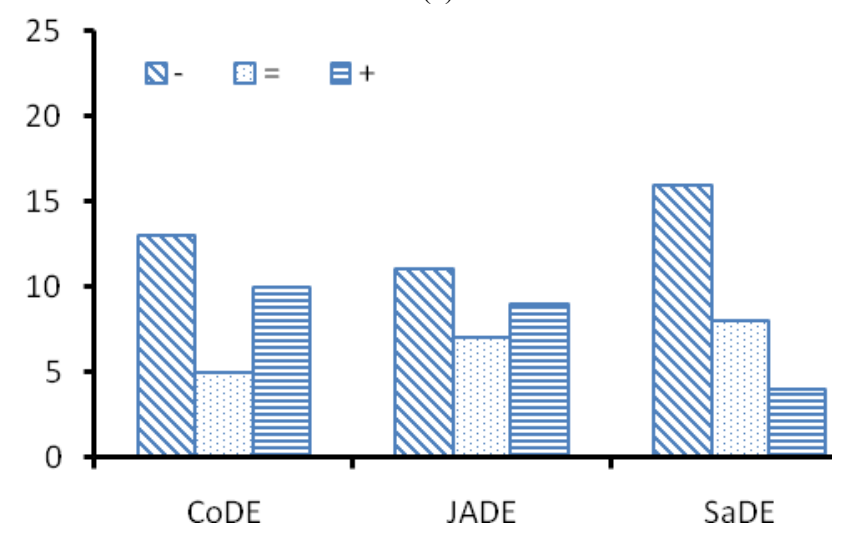

(c)

Fig. 13 The statistical significance results of comparison between EDA-FL and other EAs on 10-D(a), 30-D(b) and 50-D(c) problems respectively.

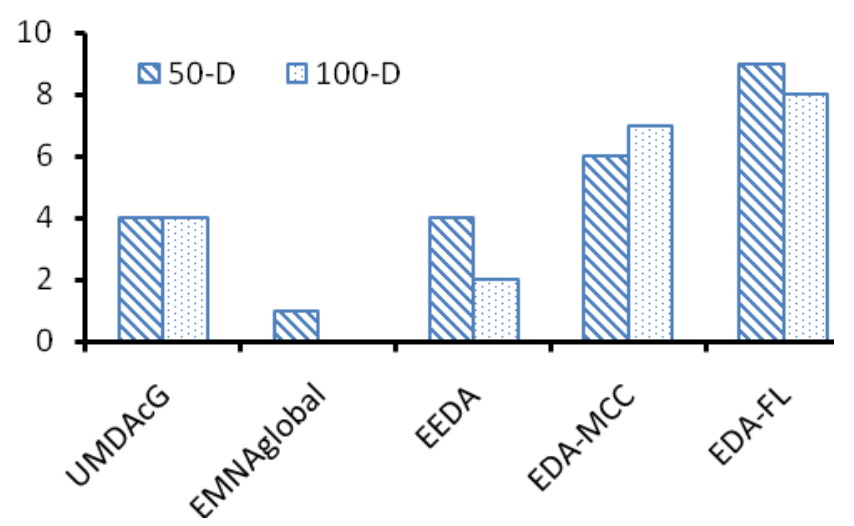

Fig. 14 The number of cases on which each algorithm performs the best in the comparison between EDA-FL and other EDAs.

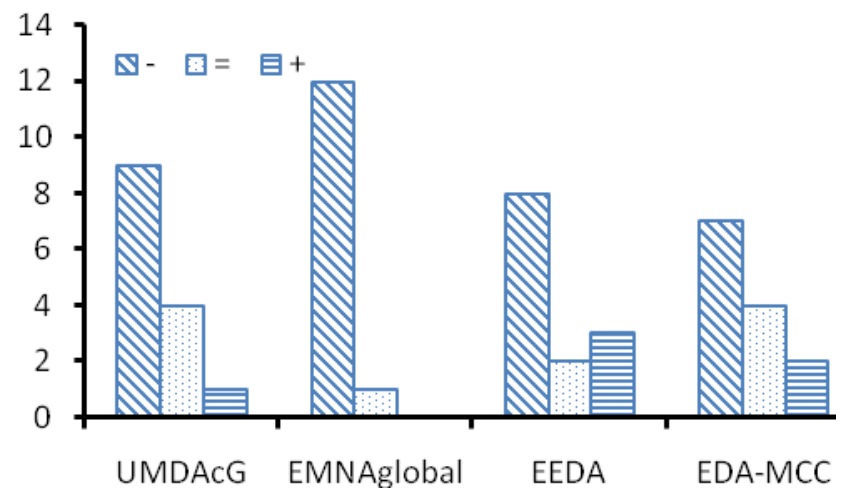

(a)

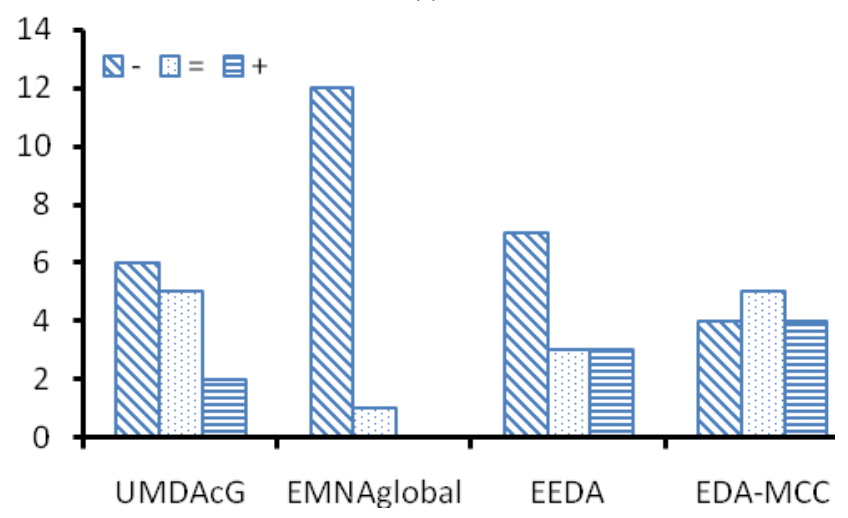

(b)

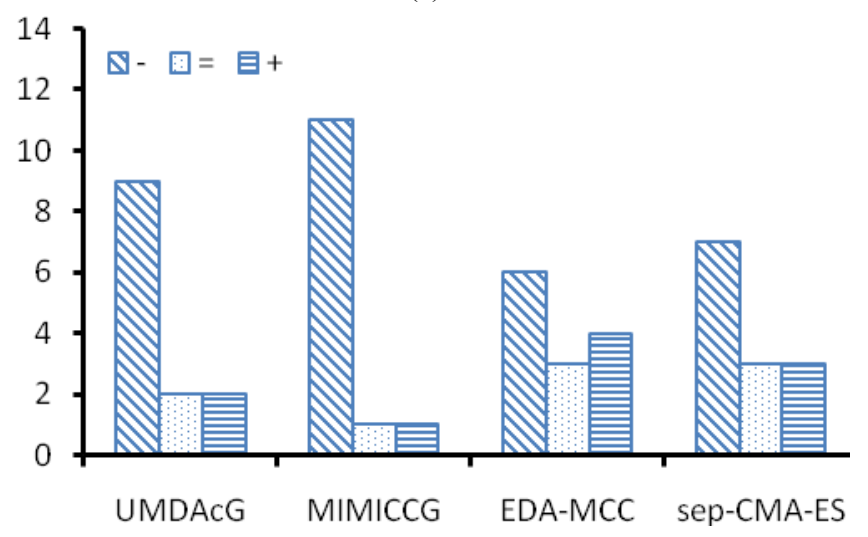

(c)

Fig. 15 The statistical significance results of comparison between EDA-FL and other EDAs on 50- $D(\mathrm{a}), 100-D(\mathrm{~b})$ and $500-D(\mathrm{c})$ problems respectively. 
3) Experiment series 3 (comparison with other EDAs and further algorithms for the scaling-up benchmark functions with 50/100/500 dimension):

Four algorithms are involved in experimental comparisons for 50D/100D/500D: $\mathrm{UMDA}_{\mathrm{c}}^{\mathrm{G}}$ [5], EMNA global [5], EEDA [6], and EDA-MCC [12]. $\mathrm{UMDA}_{\mathrm{c}}{ }^{\mathrm{G}}$ is a univariate Gaussian EDA, and EMNA $A_{\text {global }}$ is multivariate Gaussian EDA. Because the two EDAs have been studied theoretically and experimentally and applied in real-world, it makes sense comparing them with our proposed algorithm. EDA-MCC has been proposed to solve the large-scale optimization problem, so it is significant to be compared. Two other algorithms, $\mathrm{MIMIC}_{\mathrm{c}}{ }^{\mathrm{G}}$ and sep-CMA-ES are included in the comparison for 500-D, because they have good performance for high dimension problems [12]. The means and standard deviations for 10 runs are presented in TABLE VIII, $\mathrm{X}$ and XI, which can be found in the supplementary file of the paper. The best result (with the minimal mean value) is bolded in each row. Fig. 14 shows that the number of cases on which each algorithm performs the best in the comparison between EDA-FL and other EDAs. And the statistical significance results are summarized in Fig. 15.

Result analysis - With great exploitation ability, our proposed algorithm has great advantages on the unimodal function $F_{1}-F_{5}$. Again, the algorithm gives clearly better results than the other algorithms in high dimension $(500-D)$. The results indicate that our proposed algorithm is effective.

\section{CONCLUSION}

In this paper, we have proposed a new estimation of distribution algorithm with filtering and learning (EDA-FL) to solve non-linear continuous optimization problem. First, we propose a new EDA framework incorporating filtering where the idea of Kalman filtering is adopted to modify some individuals, which in turn affect the model, using an estimation error matrix from historic information. Then, a sampling strategy with learning is proposed. Using this strategy, the decision on where to sample is made according to the statistical information about the objective values of sampled individuals to avoid completely random sampling. Computational experiments on a collection of general benchmark functions with different dimensions demonstrate that the EDA-FL is effective.

Given the promising results of introducing the filtering and learning mechanisms in EDA, further research could be done to improve the filtering operations and the learning strategy. Another direction could be to apply the new EDA to problems in practice and problems with discrete variables.

\section{References}

[1] L. X. Tang, C. Liu, J. Y. Liu, and X. P. Wang. "An Estimation of Distribution Algorithm with Resampling and Local Improvement for an Operation Optimization Problem in Steelmaking Process," IEEE Trans. Syst., Man., Cybern.: Syst., 2020. DOI: 10.1109/TSMC.2019.2962880.

[2] C. Liu, L. X. Tang, J. Y. Liu, and Z. H. Tang. A Dynamic Analytics Method Based on Multistage Modeling for a BOF Steelmaking Process. IEEE Trans. Auto. Sci. Eng., vol. 16, no. 3, pp. 1097-1109, 2019.
[3] C. Liu, L. X. Tang, and J. Y. Liu. "A Stacked Autoencoder with Sparse Bayesian Regression for End-Point Prediction Problems in Steelmaking Process," IEEE Trans. Auto. Sci. Eng., vol. 17, no. 2, pp. 550-561, 2020.

[4] H. Mühlenbein and G. Paaß, "From Recombination of Genes to the Estimation of Distribution," Binary parameters. Lecture notes in computer science 1411: parallel problem solving from nature, PPSN, 1996, pp. 178-187.

[5] P. Larrñaga and J. Lozano, "Estimation of Distribution Algorithms: A New Tool for Evolutionary Computation," Boston, MA, USA: Kluwer Academic, 2002.

[6] M. Wagner, A. Auger, and M. Schoenauer, "EEDA: A New Robust Estimation of Distribution Algorithm," Rapport de Recherche (Res. Rep.) RR-5190, INRIA, 2004.

[7] M. Gallagher, M. Frean, and T. Downs, "Real-valued Evolutionary Optimization Using a Flexible Probability Density Estimator," in Proc. GECCO, 1999, pp. 840-846.

[8] P. Bosman and D. Thierens, "Advancing Continuous IDEAs with Mixture Distributions and Factorization Selection Metrics," in Proc. Optimization Building Using Probabilistic Models OBUPM Workshop GECCO, 2001, pp. 208-212.

[9] Q. Lu and X. Yao, "Clustering and Learning Gaussian Distribution for Continuous Optimization," IEEE Trans. Syst., Man, Cybern. C, Appl. Rev., vol. 35, no. 2, pp. 195-204, 2005.

[10] Y. S. Liang, Z. G. Ren, X. H. Yao, Z. R. Feng, A. Chen, and W. H. Guo, "Enhancing Gaussian Estimation of Distribution Algorithm by Exploiting Evolution Direction With Archive," IEEE Trans. Cybern., vol. 50, no. 1, pp. 140-152, 2020.

[11] J. Y. Sun, Q. F. Zhang, and E. P. K. Tsang, "DE/EDA: A New Evolutionary Algorithm for Global Optimization," Inform. Sciences, vol. 169, pp. 249-262, 2005.

[12] W. S. Dong, T. S. Chen, P. Tino, and X. Yao, "Scaling Up Estimation of Distribution Algorithms for Continuous Optimization," IEEE Trans. Evol. Comput., vol. 17, pp. 797-822, 2014.

[13] S. Tsutsui, M. Pelikan, and D. Goldberg, "Evolutionary Algorithm Using Marginal Histogram Models in Continuous Domain," in Proc. Optimization Building Using Probabilistic Models OBUPM Workshop GECCO, 2001, pp. 230-233.

[14] B. Yuan and M. Gallagher, "Playing in Continuous Spaces: Some Analysis and Extension of Population-Based Incremental Learning," in Proc. IEEE Congr. Evol. Comput., vol. 1, pp. 443-450, 2003.

[15] N. Ding, S. Zhou, and Z. Sun, "Optimizing Continuous Problems Using Estimation of Distribution Algorithm Based on Histogram Model," in Proc. 6th Conf. Simu. Evol. Learning, 2006, pp. 545-552.

[16] N. Ding, S. Zhou, H. Zhang, and Z. Sun, "Marginal Probability Distribution Algorithm: A Competent Method for Continuous Optimization," J. Comput. Sci. Technol., vol. 23, no. 1, pp. 35-43, 2008.

[17] A. Zhou, J. Y. Sun, and Q. F. Zhang,"An Estimation of Distribution Algorithm With Cheap and Expensive Local Search Methods," IEEE Trans. Evol. Comput., vol. 19, no. 6, pp. 807-822, 2015.

[18] P. Bosman and D. Thierens, "Numerical Optimization with Real-Valued Estimation-of-Distribution Algorithms," In Scalable Optimization via Probabilistic Modeling: From Algorithms to Applications, M. Pelikan,K. Sastry, and E. Cantu-Paz, Eds. Berlin/Heidelberg, Germany: SpringerVerlag, 2006, pp. 91-120.

[19] H. Mühlenbein, T. Mahnig, and A. Ochoa. "Schemata, Distributions and Graphical Models in Evolutionary Optimization," J. Heuristics, vol. 5, no. 2, pp. 213-247, 1999.

[20] R. Etxeberria and P. Larrañaga. "Global Optimization Using Bayesian Networks," In Second Symp. Artif. Intell. (CIMAF-99), 1999, pp. 332-399.

[21] S. Shakya and J. McCall. "Optimization by Estimation of Distribution with DEUM Framework Based on Markov Random Fields," Inter. J. Auto. Comput., vol. 4, no. 3, pp. 262-272, 2007.

[22] M. Pelikan and DE. Goldberg. "Genetic Algorithms, Clustering, and the Breaking of Symmetry," In Parallel Problem Solving from Nature-PPSN VI 6th International Conference, 2007, pp. 385-394.

[23] R. Santana, P. Larrañaga, and JA. Lozano. "Mixtures of Kikuchi Approximations," In Proc. 17th Eur. Conf. Mach. Learn.: ECML 2006, volume 4212 of Lecture Notes in Artificial Intelligence, 2006, pp. $365-376$. 
[24] W. S. Shao, D. C. Pi, and Z. S. Shao, "A Pareto-Based Estimation of Distribution Algorithm for Solving Multiobjective Distributed No-Wait Flow-Shop Scheduling Problem With Sequence-Dependent Setup Time," IEEE Trans. Auto. Sci. Eng., vol. 16, no. 3, pp. 1344-1360, 2019.

[25] W. Shi, W. N. Chen, Y. Lin, T. L. Gu, S. Kwong, and J. Zhang, "An Adaptive Estimation of Distribution Algorithm for Multipolicy Insurance Investment Planning," IEEE Trans. Evol. Comput., vol. 23, no. 1, pp. $1-14,2019$.

[26] D. Pfeifer and J. Nešlehová, "Modeling Dependence in Finance and Insurance: the Copula Approach,". Blätter DGVFM 26, 805 ,2004.

[27] R. B. Nelsen,"An Introduction to Copulas," Lecture Notes in Statistics 139, Springer, N.Y., 1999.

[28] L. F. Wang, J. C. Zeng, and Y. Hong, "Estimation of Distribution Algorithm Based on Copula Theory," In IEEE Cong. Evol. Comput., 2009.

[29] Y. Gao, "Multivariate Estimation of Distribution Algorithm with Laplace Transform Archimedean Copula," In Inter. Conf. Inf. Eng. Comput. Sci., 2009.

[30] L. F. Wang, X. D. Guo, J. C. Zeng, and Y. Hong, "Using Gumbel Copula and Empirical Marginal Distribution in Estimation of Distribution Algorithm," In Third Inter. Workshop Advanced Comput. Intell., 2010.

[31] A. Cuesta-Infante, R. Santana, J. I. Hidalgo, C. Bielza, and P. Larraňaga, "Bivariate Empirical and n-Variate Archimedean Copulas in Estimation of Distribution Algorithms," In IEEE Cong. Evol. Comput., 2010.

[32] B. L. Ye, H. Gao, X. P. Wang, and J. C. Zeng, "Estimation of Distribution Algorithm Based on Nested Archimedean Copulas Constructed with Lévy subordinators," In IEEE 11th Inter. Conf. Comput.-Aided Indus. Design \& Concept. Design, 2010.

[33] Y. Gao, X. Hu, and H. L. Liu, "Estimation of Distribution Algorithm Based on Multivariate Gaussian Copulas," In IEEE Inter. Conf. Prog. Inf. Comput., 2010.

[34] X. D. Guo, L. F. Wang, J. C. Zeng, and X. L. Zhang, “Copula Estimation of Distribution Algorithm with PMLE," In Seventh Inter. Conf. Natural Comput., 2011.

[35] Y. Gao, L. X. Peng, F. F. Li, M. Liu, and X. Hu, "Pareto-Based Multi-objective Estimation of Distribution Algorithm with Gaussian Copulas and Application in RFID Network Planning," In IEEE fifth Inter. Conf. Advanced Comput.Intell., 2012 (ICACI).

[36] H. D. de Mello Jr, A. V. A. da Cruz, and M. M. B. R. Vellasco, "Estimation of Distribution Algorithm Based on a Multivariate Extension of the Archimedean Copula," In BRICS Cong. Comput. Intell. \& 11th Brazilian Cong. Comput. Intell., 2013.

[37] H. D. Xu, M. Y. Jiang, and K. Xu, "Archimedean Copula Estimation of Distribution Algorithm Based on Artificial Bee Colony Algorithm," $J$. Syst. Eng. Elec., vol. 26, no. 2, pp. 388-396, 2015.

[38] M. Hyrŝ and J. Schwarz, "Advanced Parallel Copula Based EDA," In IEEE Symp. Series Comput. Intell., 2016.

[39] H. D. de M. Junior, L. Mart, A. V. A. da Cruz, and M. M. B. R. Vellasco, "Evolutionary Algorithms and Elliptical Copulas Applied to Continuous Optimization Problems," Inf. Sci., vol. 369, pp. 419-440, 2016.

[40] S. J. Gao, Clarence, and C. W. de Silva, "Estimation Distribution Algorithms on Constrained Optimization Problems," Appl. Math. Comput., vol. 339, pp. 323-345, 2018.

[41] B. Qian, Z. C. Li, and R. Hu, "A Copula-based Hybrid Estimation of Distribution Algorithm Form-Machine Reentrant Permutation Flow-shop Scheduling Problem," Appl. Soft Comput., vol. 61, pp. 921-934, 2017.

[42] C. Liu, H. P. Chen, R. Xu, and Y. Wang, "Minimizing the Resource Consumption of Heterogeneous Batch-Processing Machines Using a Copula-Based Estimation of Distribution Algorithm," Appl. Soft Comput. J., vol. 73, pp. 283-305, 2018.

[43] R. E. Kalman. "A New Approach to Linear Filtering and Prediction Problems,"Trans. ASME-J. Basic Eng., pp. 35-45, 1960.

[44] A. Muruganantham and K. C. Tan, "Evolutionary Dynamic Multiobjective Optimization Via Kalman Filter Prediction," IEEE Trans. Cybern., vol. 46, no. 12, pp. 2862-2873, 2016.

[45] J. Strelen and F. Nassaj, "Analysis and Generation of Random Vectors with Copulas," In Proc. Winter Simu. Conf., 2007, pp. 488-496,.

[46] W. B. Powell and P. Frazier, "Optimal Learning," Tutor. Oper. Res., 2008, pp. 213-246.
[47] P. Ge, Y. Meng, J. Liu, L. Tang, and Ren Zhao, "Logistics Optimisation of Slab Pre-marshalling Problem in Steel Industry", to appear in International Journal of Production Research, available online 2019, DOI: 10.1080/00207543.2019.1641238

[48] S. S. Gupta and K. J. Miescke. "Bayesian Look Ahead One-stage Sampling Allocations for Selection of the Best Population," J. Stat. Plan. Inf., vol. 54, pp. 229-244, 1996.

[49] J. J. Liang, B. Y. Qu, P. N. Suganthan, and Alfredo G. Hernández-Díaz, "Problem Definitions and Evaluation Criteria for the CEC 2013 Special Session on Real-parameter Optimization," 2013. [Online]. Available: http://www.ntu.edu.sg/home/ epnsugan/

[50] P. Suganthan, N. Hansen, J. Liang, K. Deb, Y. Chen, A. Auger, and S. Tiwari. "Problem Definitions and Evaluation Criteria for the CEC 2005 Special Session on Real-parameter Optimization," 2005, Tech. Rep.[Online]. Available: http://www.ntu.edu.sg/home/EPNSugan/

[51] X. Yao, Y. Liu, and G. Lin, "Evolutionary Programming Made Faster,"IEEE Trans. Evol. Comput., vol. 3, no. 2, 1999, pp. 82-102.

[52] L. X. Tang, Y. Dong, and J. Y. Liu, "Differential Evolution with an Individual-Dependent Mechanism," IEEE Trans. Evol. Comput., vol. 19, no. 4, pp. 560-574, 2015.

[53] Y. Wang, Z. Cai, and Q. Zhang, "Differential Evolution with Composite Trial Vector Generation Strategies and Control Parameters", IEEE Trans. Evol. Comput., vol. 15, no. 1, pp. 55-66, 2011.

[54] M. G. Epitropakis, D. K. Tasoulis, N. G. Pavlidis, V. P. Plagianakos, and M. N. Vrahatis, "Enhancing Differential Evolution Utilizing Proximity-based Mutation Operators," IEEE Trans. Evol. Comput., vol. 15, no. 1, pp. 99-119, 2011.

[55] A. K. Qin, V. L. Huang, and P. N. Suganthan, "Differential Evolution Algorithm with Strategy Adaptation for Global Numerical Optimization," IEEE Trans. Evol. Comput., vol. 13, no. 2, pp. 398-417, 2009.

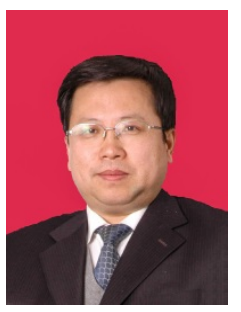

Lixin Tang (M'09-SM'14) received the B.Eng. degree in industrial automation, the M.Eng. degree in systems engineering and the Ph.D. degree in control theory and application from Northeastern University, Shenyang, China, in 1988, 1991, 1996, respectively.

He is a Fellow of Chinese Academy of Engineering, a Chair Professor of the Institute of Industrial \& Systems Engineering, Northeastern University; the Director of Key Laboratory of Data Analytics and Optimization for Smart Industry (Northeastern University), Ministry of Education, China; and the Head of Centre for Artificial Intelligence and Data Science.

His research interests cover industrial big data science, data analytics and machine learning, reinforcement learning and dynamic optimization, computational intelligent optimization, plant-wide production and logistics planning, production and logistics batching and scheduling and engineering applications in manufacturing (steel, petroleum-chemical, nonferrous), energy, resources industry and logistics systems. His research papers have appeared in academic journals such as Operations Research, IIE Transactions, Naval Research Logistics, IEEE Transactions on Evolutionary Computation, IEEE Transactions on Power Systems, IEEE Transactions on Control Systems Technology, and European Journal of Operational Research. 
He serves as an Associate Editor of IISE Transactions, IEEE Transactions on Evolutionary Computation, IEEE Transactions on Cybernetics, Journal of Scheduling, International Journal of Production Research, Journal of the Operational Research Society, in Editorial Board of Annals of Operations Research, and an Area Editor of the Asia-Pacific Journal of Operational Research.

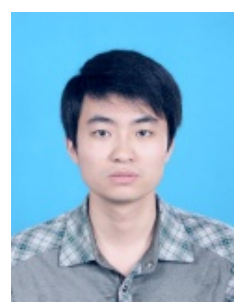

Xiangman Song is a Ph.D. candidate in logistics optimization and control at the Institute of Industrial and Systems Engineeringin Northeastern University, Shenyang, China.

His research interests include operations analytics and optimization for smart industry, optimal and predictive control, data analytics and machine learning, and computational intelligent optimization.

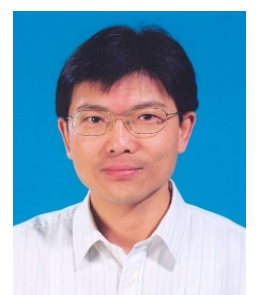

Jiyin Liu received the B.Eng. degree in industrial automation and the M.Eng. degree in systems engineering from Northeastern University, Shenyang, China, in 1982 and 1985, respectively, and the Ph.D. degree in manufacturing engineering and operations management from the University of Nottingham, Nottingham, U.K., in 1993.

Currently he is Professor of Operations Management in the School of Business and Economics at Loughborough University, Leicestershire, U.K. He is also a Cheung Kong Scholars Visiting Chair Professor at the Institute of Industrial and Systems Engineering, Northeastern University, China. His research interests are in operations planning and scheduling problems in production, logistics and supply chains and in mathematical modeling, optimization and heuristic methods. $\mathrm{He}$ has published papers in journals such as the European Journal of Operational Research, IEEE Transactions, IIE Transactions, International Journal of Production Research, Naval Research Logistics, Operations Research, and Transportation Research.

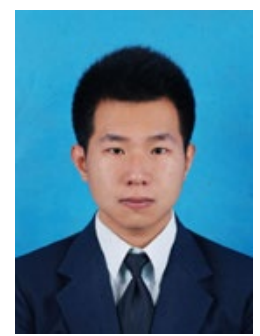

Chang Liu is currently pursuing the Ph.D. degree in systems engineering with the Institute of Industrial and Systems Engineering, Northeastern University, Shenyang, China.

His research interests include data analytics and optimization for smart industry, model predictive control, machine learning, computational intelligent optimization, and engineering applications in various industrial processes. He has published several papers in journals such as IEEE Transactions on Systems, Man, and Cybernetics: Systems, IEEE Transactions on Automation Science and Engineering, and Neurocomputing. 